\title{
A Thermodynamic Study of the Ternary System, Triethylamine, Water and Heavy Water, with Special Reference to Isotope Separation Problems
}

\author{
C. U. LI N D ER S T R $\emptyset \mathrm{M}-\mathrm{L}$ A N G
}

The Chemistry Department, The Danish Atomic Energy Commission, Ris $\phi$, Denmark

1. An experimental isotope separation and solubility study in the two-phase region of the system, water $\left(\mathrm{D}_{2} \mathrm{O}+\mathrm{H}_{2} \mathrm{O}\right)-$ triethylamine (TEA) has been carried out.

Concentrations of $\mathrm{D}_{2} \mathrm{O}$ and $\mathrm{H}_{2} \mathrm{O}$ in TEA have been determined by their absorption at $4.0 \mu$ and $6.1 \mu$, respectively.

2. Diethylamine and ethylamine as impurities in the triethylamine used, have been determined by quantitative paper chromatography using a modified Levy-procedure.

3. Fundamental thermodynamic equations pertinent to the calculation of hydrogen isotope separation effects in the system at equilibrium are developed.

4. The total water concentration, $x_{\mathrm{w}}$, of a phase and the isotopic composition, $y_{\mathrm{D}}$, of this water (both in mole fractions) are chosen as independent variables. It is presumed that changes in activity coeff $\mathrm{i}$ cients, $f$, when $y_{\mathrm{D}}$ is varied at constant $x_{\mathrm{w}}$ are small compared to changes when $x_{\mathrm{w}}$ is varied at constant $y_{\mathrm{D}}$.

On this basis it is postulated that reasonable first-order or secondorder approximate relations are obtained when it is assumed that $\left(\partial \ln f / \partial y_{\mathrm{D}}\right)_{x_{\mathrm{w}}, T}=0$ or $\left(\partial \ln f / \partial y_{\mathrm{D}}\right)_{x_{\mathrm{w}}, T}=$ a constant $(i . e$. independent of $\left.y_{\mathrm{D}}\right)$, respectively.

5. These relations are introduced into the fundamental equations and it is shown that in both cases a numerical calculation of the isotope separation effect can be carried through with reasonable accuracy, using available enthalpy data and/or vapour pressure data for pure $\mathrm{H}_{2} \mathrm{O}$-TEA together with two-phase equilibrium data for the binary systems, $\mathrm{H}_{2} \mathrm{O}$-TEA and $\mathrm{D}_{2} \mathrm{O}$-TEA.

The numerical calculation indicates that $\partial \ln f / \partial y_{\mathrm{D}}$ is less than $0.01 \times\left(\partial \ln f / \partial x_{\mathrm{w}}\right)$.

6. The relative solubility change of water in TEA, when $\mathrm{H}_{2} \mathrm{O}$ is replaced by $\mathrm{D}_{2} \mathrm{O}$ at constant temperature, ( $\mathrm{d} \ln x^{\prime}{ }_{\mathrm{w}} / \mathrm{d} y_{\mathrm{D}}^{\prime}$ ) eq, $T$ multiplied by a factor, $Q=1+x^{\prime}{ }_{\mathrm{w}}\left(\partial \ln f^{\prime}{ }_{\mathrm{w}} / \partial x_{\mathrm{w}}\right)$, expressing the degree of ideality in the triethylamine phase, is found to be a simple and convenient measure of the isotope separation effect.

It is shown that the large $\mathrm{d} \ln x^{\prime}{ }_{\mathrm{w}} / \mathrm{d} y^{\prime}{ }_{\mathrm{D}}$ found in this system and in other similar systems must be compensated to a great extent by a small $Q$, so the absence of any high isotope separation effect is easily understood. 
$\mathrm{T}^{\mathrm{T}}$ he remarkably high difference between the solubility of ordinary water $\left(\mathrm{H}_{2} \mathrm{O}\right)$ and heavy water $\left(\mathrm{D}_{2} \mathrm{O}\right)$ in triethylamine (TEA) may seem at first glance to promise a high isotope separation effect when $\mathrm{H}_{2} \mathrm{O}$ and $\mathrm{D}_{2} \mathrm{O}$ are mixed. However, Carlson ${ }^{1}$, using water enriched with a few percent $\mathrm{D}_{2} \mathrm{O}$, found hardly any effect, (for instance, at $35^{\circ} \mathrm{C}$ the separation factor was $1.004 \pm 0.004$ ) and Hahn and Street ${ }^{2}$ working with $98 \% \mathrm{D}_{2} \mathrm{O}$ found no effect at $15-25^{\circ} \mathrm{C}$.

The high expectation probably is based on the often applicable assumption that the solubility of one component in another to a first approximation is independent of the addition of a third component, a condition which, however, is far from being fulfilled when the third component (e.g. heavy water), as is the case here, is soluble in both phases.

In order to corroborate these results an experimental study has been undertaken and the system has been given a theoretical treatment. The discussion below is based on elementary thermodynamic principles which, however, do not seem to have been applied to this particular problem before; therefore the solution may have some general interest.

Approximate chemical potential functions are developed and a numerical calculation of the separation effects based on available thermodynamic data is attempted. The resulting figures may furnish only the order of magnitude of the terms involved, however, this appears sufficient to illustrate their relative importance.

\section{EXPERIMENTAL PART}

The triethylamine was a Fluka p.a. quality dried for a short time with sodium and decanted.

The heavy water was a $99.8 \%$ quality from which a $20 \% \mathrm{D}_{2} \mathrm{O}$ standard solution was prepared.

Procedure. In equilibration tubes shaped as shown on Fig. $1,3 \mathrm{ml}$ of triethylamine were placed in the horizontal part together with varying amounts (from 0.2 to $1 \mathrm{ml}$ ) of $\mathrm{H}_{2} \mathrm{O}$, pure $\mathrm{D}_{2} \mathrm{O}$, or the $20 \%$ mixture. The tubes were shaken in a thermostat (regulating to within $0.01^{\circ} \mathrm{C}$ ). A mercury seal was placed on top of the ground joints.

The shaking was fairly slow and it was extended over night as a convenient routine step though of course much shorter periods would have been sufficient. In order to detect any slow exchange with TEA or impurities in it, even longer periods were used.

After equilibration the mixture, while in the thermostat, was transferred to the vertical section of the tube and the mercury poured off.

A $2 \mathrm{ml}$ sample of the TEA phase was taken and, if necessary for the subsequent determination, diluted with dry TEA.

Then a sample (either $50 \mu \mathrm{l}$ or $100 \mu \mathrm{l}$ ) of the water phase was drawn with a constriction pipette (Carlsberg). It was noticed that even a few bubbles of expired air through the TEA phase, when passing it with the pipette were enough to produce a turbidity - undoubtedly a carbon dioxide effect. No measures were taken in order to reduce the amount of $\mathrm{CO}_{2}$ present but as the equilibration procedure was always the same, the relative solubility was probably not affected, nor was the change of solubility with temperature.

The water phase samples were diluted with dry TEA $(2-3 \mathrm{ml})$. In this way the $\mathrm{D} / \mathrm{H}$ ratio in the two phases became directly comparable.

The concentrations were determined with a "Perkin Elmer 221" infrared spectrophotometer, " $\mathrm{H}_{2} \mathrm{O}$ " at $6.1 \mu$ and " $\mathrm{D}_{2} \mathrm{O}$ " at $4.0 \mu$, in $0.125 \mathrm{~mm} \mathrm{CaF}{ }_{2}$-cells. $20 \% \mathrm{D}_{2} \mathrm{O}$ for the equilibration was chosen because in this concentration region the ratio of the optical densities at the two wavelengths was near unity (approx. 1.2). An appropriate number of standards was always run simultaneously with the unknowns. It was found impossible to store standards in a satisfactory way, so fresh ones were always made up for each experiment.

Acta Chem. Scand. 16 (1962) No. 7 


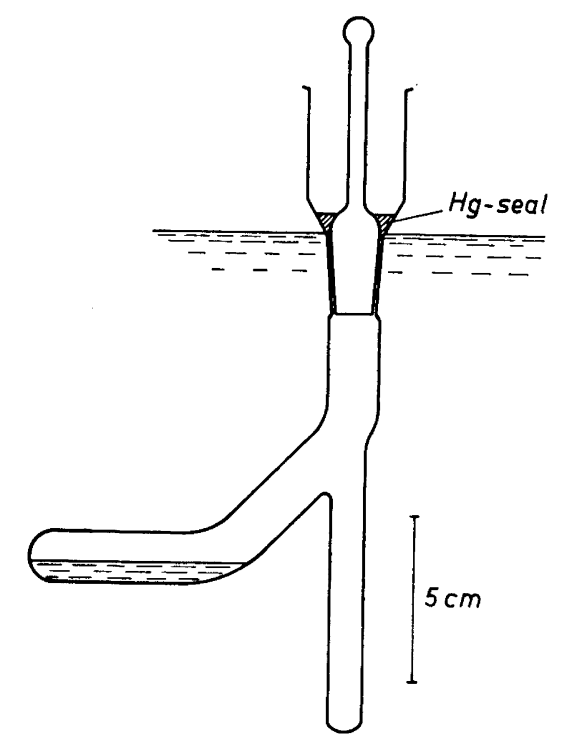

Fig. 1. Equilibration tube.

The varying amount of water phase revealed to what extent disturbing impurities accumulated in the water. The only other source of error that seemed likely to have any importance was a rapid exchange with an impurity in the TEA.

The supplier gave the information that it might contain some diethylamine (DEA) and ethanol. The actual amounts of DEA and, as it turned out, monoethylamine (MEA) present were determined by using a modified Levy procedure ${ }^{10}$, i.e. reaction with dinitrofluorobenzene and identification and estimation by chromatography on paper. A somewhat higher $\mathrm{pH}$ than Levy's was used in order to make the reaction conveniently fast. The DNP derivatives were extracted with ether after neutralizing the reaction mixture to $\mathrm{pH} 5-6$. Dinitrophenol was removed from the ether phase with aquous sodium hydroxide. A $0.1 \mathrm{M}$ phosphate buffer $\mathrm{n} 50 \%$ ethanol was used as solvent for the chromatographic development; however it is not claimed that the choice is ideal. Otherwise Levy's procedure was followed. The values obtained are believed to be accurate to within $10 \%$ (see Table 1).

Alcohol in TEA was estimated by oxidation for two days with $0.1 \mathrm{~N}$ potassium dichromate in $4 \mathrm{~N}$ sulphuric acid at $40^{\circ} \mathrm{C}$ and determination of the excess of dichromate by reaction with iodide and titration of the iodine formed with thiosulphate. The value obtained is believed to be accurate to within $10 \%$ (see Table 1).

TEA, dried for a short time, i.e. until bubbles ceased to be evolved, with sodium showed no decrease in alcohol content (tested at different alcohol concentrations) nor was there any detectable residue after evaporation in vacuum.

\section{EXPERIMENTAL RESULTS}

There is a slow chemical exchange between $20 \% \mathrm{D}_{2} \mathrm{O}$ and TEA which leads to a small decrease in $\mathrm{D}_{2} \mathrm{O}$ concentration. It amounts to about $1-2 \%$ (relative) per day. It may be assumed therefore that, if the total amount of water present in an equilibration experiment is $3 / 2$ times the water dissolved in the TEA phase the expected recovery will be approx. $99 \%$. In most experiments, however, the amount of water is much larger and therefore the correc- 
Table 1. Hydrogen exchanging impurities in the triethylamine used, as found by the analytical procedures described in the text.

\author{
Diethylamine \\ Ethylamine \\ Ethanol \\ Total
}

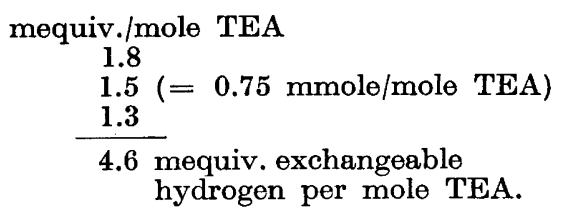

tion much smaller. In agreement with this, the simple recovery average of 21 experiments, corrected for rapid exchange (see below) was $99.6 \%$ (S.D. of the distribution $1 \%$ ). Equilibrium is probably attained in much less than one day, so in no case has a correction to the distribution ratio to be made.

Variation of the ratio of water phase to TEA phase had no effect on the optical density ratio of the water phase; therefore, no impurities affecting the spectrophotometric determination appear to accumulate in the water phase.

If a rapidly exchanging impurity is present it may react in the following manner

$$
\frac{1}{2} \mathrm{D}_{2} \mathrm{O}+\mathrm{IH} \rightleftharpoons \frac{1}{2} \mathrm{H}_{2} \mathrm{O}+\mathrm{ID}
$$

the equilibrium constant being

$$
E=\frac{[\mathrm{ID}]\left[\mathrm{H}_{2} \mathrm{O}\right]^{\frac{1}{2}}}{[\mathrm{IH}]\left[\mathrm{D}_{2} \mathrm{O}\right]^{\frac{1}{2}}} \simeq 1
$$

If ID $\equiv 2 \varepsilon$ (in moles per mole TEA-phase) and $y_{\mathrm{D}} /\left(1-y_{\mathrm{D}}\right) \equiv r$, where $y_{\mathrm{D}}$ is mole fraction of $\mathrm{D}_{2} \mathrm{O}$, i.e. $y_{\mathrm{D}} \simeq 0.20$ (see eqn. 8 ), then

$$
\Delta r=\frac{W y_{\mathrm{D}}-\varepsilon}{W\left(1-y_{\mathrm{D}}\right)+\varepsilon}-\frac{W y_{\mathrm{D}}}{W\left(1-y_{\mathrm{D}}\right)} \simeq-\frac{\varepsilon}{\left(1-y_{\mathrm{D}}\right)^{2} W}
$$

Here $\left(1-y_{\mathrm{D}}\right)=\frac{4}{5}$ so $\Delta r=-\frac{25}{16} \frac{\varepsilon}{W}$. W is the number of moles of water $\left(20 \% \mathrm{D}_{2} \mathrm{O}\right)$ in equilibrium with, and contained in, one mole of TEA phase (and therefore approx. one mole of TEA). $\varepsilon$ is independent of $W$ as long as the amount of impurity is small compared to the amount of $\mathrm{D}_{2} \mathrm{O}$.

The $r$-values of the water phase samples need no correction for rapidly exchanging impurity, because the standards run simultaneously are treated in exactly the same manner - provided, as already mentioned, the impurities do not accumulate in the water phase and there interfere with the measurements.

The TEA phase $r$-values will be too high when compared to ordinary standards in which the $\mathrm{D}_{2} \mathrm{O}$ concentration $\left(y_{\mathrm{D}}\right)$ has been somewhat reduced by the impurities. Relevant standards, which were prepared from TEA "deuterated" with $20 \% \mathrm{D}_{2} \mathrm{O}$ and then dried, gave lower TEA phase $r$-values in agreement with the above; however, a comparison of the different standard curves obtained gave only fair agreement with theory even when this was elaborated to take into account corrections based on: (1) distribution factors 
Table 2. Experimental results. $3 \mathrm{ml}$ triethylamine were equilibrated with varying amounts of $20 \% D_{2} \mathrm{O}$ (i.e., $y_{\mathrm{D}}=0.20$ ). The isotopic composition of samples drawn from the water phase (and diluted with TEA) and from the TEA phase was determined spectrophotometrically at $6.1 \mu$ (" $\mathrm{H}_{2} \mathrm{O}$ ") and $4.0 \mu$ (" $\mathrm{D}_{2} \mathrm{O}$ "). For details see text.

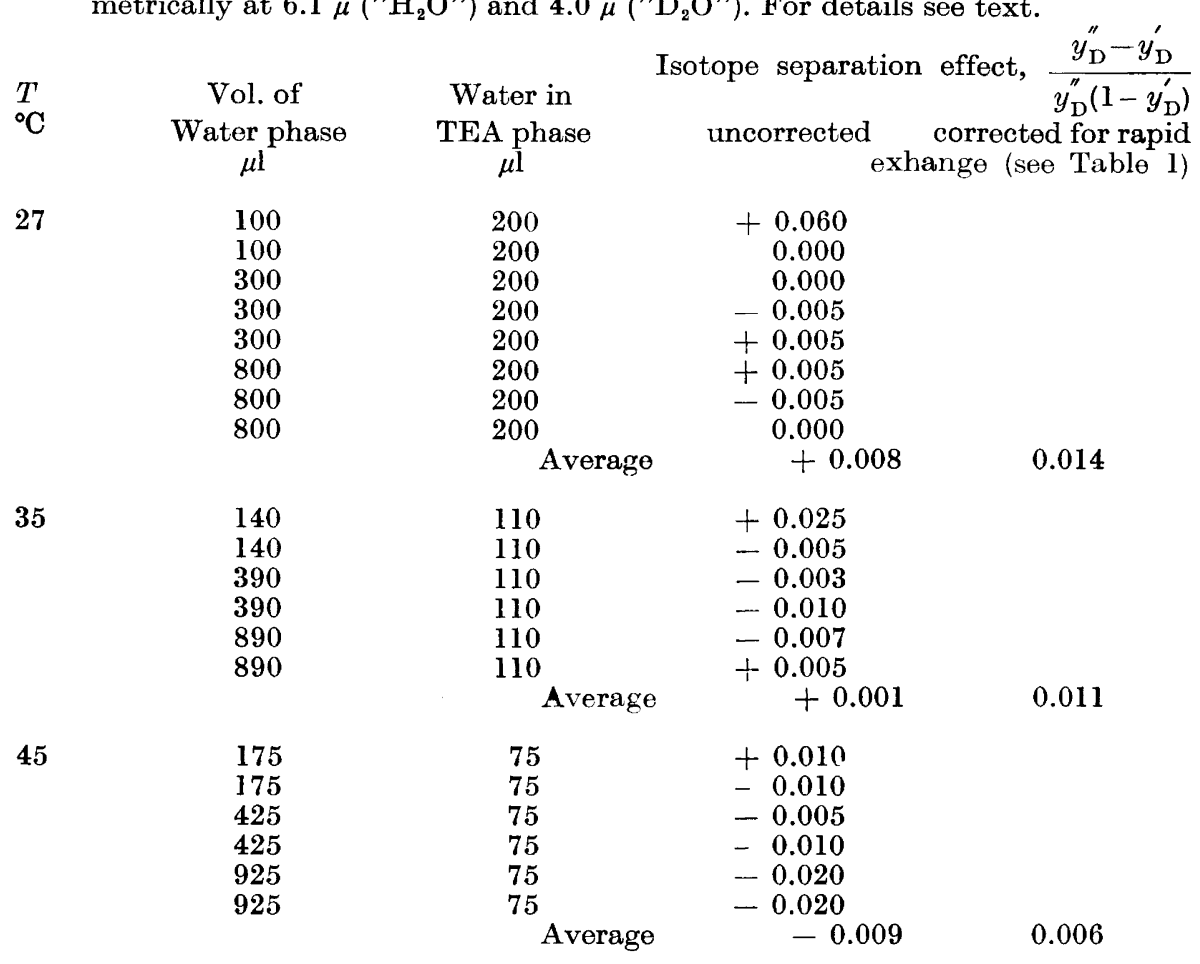

of the impurities, and (2) their absorptions at the two wavelengths. Deviations from Lambert-Beer's law, caused by shifts, with changing water concentration, in the peak wave-length at $6 \mu$, were a further complication.

In addition the reduction in TEA $r$-values was greater than accounted for by chemical analysis and though the explanation may be a trace of water present, it was felt safer to base the TEA phase $r$-values on a correction calculated from known available exchangeable hydrogen (see Table 1).

Table 2 shows the results of the analytical determinations. The isotope effect, $\left[y_{\mathrm{D}}^{\prime \prime}-y_{\mathrm{D}}^{\prime}\right] /\left[y_{\mathrm{D}}^{\prime}\left(1-y_{\mathrm{D}}^{\prime \prime}\right)\right]$, (see eqn. 9) has been calculated from the $r$-values of the TEA phase and the water phase, $r^{\prime}$ and $r^{\prime \prime}$, respectively. One has $\left[y_{\mathrm{D}}^{\prime \prime}-y_{\mathrm{D}}^{\prime}\right] /\left[y_{\mathrm{D}}^{\prime}\left(1-y_{\mathrm{D}}^{\prime \prime}\right)\right]=\left(r^{\prime \prime}-r^{\prime}\right) / r$ where $r$ with sufficient accuracy is $0.2 / 0.8=1 / 4$.

The correction for rapid exchange has been calculated as $-\frac{\Delta r}{r}=$ $=4 \cdot \frac{25}{16} \cdot \frac{1}{5} \cdot \frac{4.6}{2} \cdot \frac{10^{-3}}{W}$, where $W$ (in moles per mole TEA) now is the 
amount of water in the TEA phase sample (or rather in the corresponding standard).

The standard deviation of $\left[y_{\mathrm{D}}^{\prime \prime}-y_{\mathrm{D}}^{\prime}\right] /\left[y_{\mathrm{D}}^{\prime}\left(1-y_{\mathrm{D}}^{\prime \prime}\right)\right]$ is estimated to be $c a$. $1-1.5 \%$ (absolute) so the averaged values should be accurate to about $0.5 \%$. However, the presence of a small amount of water in the TEA employed, a possibility that cannot be excluded, may have introduced a systematic error of about the same magnitude. The solubility determinations are of course less accurate, the estimated standard deviation being $3 \%$ (relative), and, as mentioned earlier, include a systematic error due to the presence of $\mathrm{CO}_{2}$. The relative position of the two curves is thought to be relevant to the isotope effects found. The calculation is straight forward.

\section{THEORETICAL PART}

1. Equilibrium conditions, fundamental equations. If $\mu_{\mathrm{D}}^{\prime}, \mu_{\mathrm{H}}^{\prime}$ and $\mu_{\mathrm{A}}^{\prime}$ are the chemical potentials of $\mathrm{D}_{2} \mathrm{O}, \mathrm{H}_{2} \mathrm{O}$, and TEA in the TEA-rich phase and $\mu_{\mathrm{D}}^{\prime \prime}, \mu_{\mathrm{H}}^{\prime \prime}$ and $\mu_{\mathrm{A}}^{\prime \prime}$ are the corresponding potentials in the water-rich phase, then

$$
\begin{aligned}
& \mu_{\mathrm{D}}^{\prime}=\mu_{\mathrm{D}}^{\prime \prime} \\
& \mu_{\mathrm{H}}^{\prime}=\mu_{\mathrm{H}}^{\prime \prime} \\
& \mu_{\mathrm{A}}^{\prime}=\mu_{\mathrm{A}}^{\prime \prime}
\end{aligned}
$$

at equilibrium (denoted below by "eq"), or for any change in composition and temperature (and pressure)

$$
\mathrm{d} \mu^{\prime}=\mathrm{d} \mu^{\prime \prime}
$$

for any of the components. The three equations represented by eqn. 2 may be combined in the following way

$$
x_{\mathrm{D}}^{\prime \prime} \mathrm{d} \mu_{\mathrm{D}}^{\prime \prime}+x_{\mathrm{H}}^{\prime \prime} \mathrm{d} \mu_{\mathrm{H}}^{\prime \prime}+x_{\mathrm{A}}^{\prime \prime} \mathrm{d} \mu_{\mathrm{A}}^{\prime \prime}=x_{\mathrm{D}}^{\prime \prime} \mathrm{d} \mu_{\mathrm{D}}^{\prime}+x_{\mathrm{H}}^{\prime \prime} \mathrm{d} \mu_{\mathrm{H}}^{\prime}+x_{\mathrm{A}}^{\prime \prime} \mathrm{d} \mu_{\mathrm{A}}^{\prime},
$$

where $x_{\mathrm{D}}, x_{\mathrm{H}}$ and $x_{\mathrm{A}}$ are mole fractions, i.e. the number of moles of pure component, $\mathrm{D}_{2} \mathrm{O}, \mathrm{H}_{2} \mathrm{O}$, and TEA, to be added in order to produce one mole of mixture: $x_{\mathrm{D}}+x_{\mathrm{H}}+x_{\mathrm{A}}=1$.

Changes in chemical potential may be written (at constant pressure)

$$
\mathrm{d} \mu=\left(\frac{\partial \mu}{\partial z_{1}}\right)_{T, z_{3}} \mathrm{~d} z_{1}+\left(\frac{\partial \mu}{\partial T}\right)_{z_{1}, z_{3}} \mathrm{~d} T ;\left(\mathrm{d} z_{2}=0\right)
$$

where $z_{1}$ and $z_{2}$ are any two concentrations, sufficient for describing the composition of the system.

Gibbs-Duhem's equation,

$$
x_{\mathrm{D}}\left(\frac{\partial \mu_{\mathrm{D}}}{\partial z_{1}}\right)_{T, \mathbf{z}_{\mathbf{z}}}+x_{\mathrm{H}}\left(\frac{\partial \mu_{\mathrm{H}}}{\partial z_{1}}\right)_{T, z_{\mathbf{2}}}+x_{\mathrm{A}}\left(\frac{\partial \mu_{\mathrm{A}}}{\partial z_{1}}\right)_{T, \boldsymbol{z}_{\mathbf{2}}}=0
$$

Acta Chem. Scand. 16 (1962) No. 7 
and eqn. 4 combined with eqn. 3 gives

$$
\begin{aligned}
& \left(x_{\mathrm{D}}^{\prime \prime} \frac{\partial \mu_{\mathrm{D}}^{\prime \prime}}{\partial T}+x_{\mathrm{H}}^{\prime \prime} \frac{\partial \mu_{\mathrm{H}}^{\prime \prime}}{\partial T}+x_{\mathrm{A}}^{\prime \prime} \frac{\partial \mu_{\mathrm{A}}^{\prime \prime}}{\partial T}\right) \mathrm{d} T= \\
& \left(x_{\mathrm{D}}^{\prime \prime} \frac{\partial \mu_{\mathrm{D}}^{\prime}}{\partial T}+x_{\mathrm{H}}^{\prime \prime} \frac{\partial \mu_{\mathrm{H}}^{\prime}}{\partial T}+x_{\mathrm{A}}^{\prime \prime} \frac{\partial \mu_{\mathrm{A}}^{\prime}}{\partial T}\right) \mathrm{d} T+\left(x_{\mathrm{D}}^{\prime \prime} \frac{\partial \mu_{\mathrm{D}}^{\prime}}{\partial z_{1}}+x_{\mathrm{H}}^{\prime \prime} \frac{\partial \mu_{\mathrm{H}}^{\prime}}{\partial z_{1}}+x_{\mathrm{A}}^{\prime \prime} \frac{\partial \mu_{\mathrm{A}}^{\prime}}{\partial z_{1}}\right) \mathrm{d} z_{1}
\end{aligned}
$$

Eliminating $\partial \mu_{\mathrm{A}}^{\prime} / \partial z_{1}$ (Gibbs-Duhem, eqn. 5) * and introducing $\partial \mu / \partial T=-S$ (the partial molar entropy) give

$$
\begin{aligned}
& {\left[\left(x_{\mathrm{D}}^{\prime \prime}-x_{\mathrm{D}}^{\prime} \frac{x_{\mathrm{A}}^{\prime \prime}}{x_{\mathrm{A}}^{\prime}}\right) \frac{\partial \mu_{\mathrm{D}}^{\prime}}{\partial z_{1}}+\right.}\left.\left(x_{\mathrm{H}}^{\prime \prime}-x_{\mathrm{H}}^{\prime} \frac{x_{\mathrm{A}}^{\prime \prime}}{x_{\mathrm{A}}^{\prime}}\right) \frac{\partial \mu_{\mathrm{H}}^{\prime}}{\partial z_{1}}\right]\left(\frac{\mathrm{d} z_{1}}{\mathrm{~d} T}\right)_{\mathrm{eq}, s_{\mathrm{e}}}= \\
& x_{\mathrm{D}}^{\prime \prime}\left(S_{\mathrm{D}}^{\prime}-S_{\mathrm{D}}^{\prime \prime}\right)+x_{\mathrm{H}}^{\prime \prime}\left(S_{\mathrm{H}}^{\prime}-S_{\mathrm{H}}^{\prime \prime}\right)+x_{\mathrm{A}}^{\prime \prime}\left(S_{\mathrm{A}}^{\prime}-S_{\mathrm{A}}^{\prime \prime}\right)
\end{aligned}
$$
way

Two new variables, $y_{\mathrm{D}}$ and $x_{\mathrm{W}}$, are now introduced, defined in the following

$$
\begin{gathered}
y_{\mathrm{D}} \cdot x_{\mathrm{W}} \equiv x_{\mathrm{D}} \\
\left(1-y_{\mathrm{D}}\right) \cdot x_{\mathrm{W}} \equiv x_{\mathrm{H}} \\
\text { i.e., } x_{\mathrm{W}}+x_{\mathrm{A}}=1
\end{gathered}
$$

Thus the isotope separation factor, $\alpha$, may be written

$$
\alpha=\frac{y_{\mathrm{D}}^{\prime \prime}}{1-y_{\mathrm{D}}^{\prime \prime}} / \frac{y_{\mathrm{D}}^{\prime}}{1-y_{\mathrm{D}}^{\prime}}=1+\frac{y_{\mathrm{D}}^{\prime \prime}-y_{\mathrm{D}}^{\prime}}{y_{\mathrm{D}}^{\prime}\left(1-y_{\mathrm{D}}^{\prime \prime}\right)}
$$

The following two relations (cf. eqn. 7) may now be obtained

and

$$
x_{\mathrm{D}}^{\prime \prime}-x_{\mathrm{D}}^{\prime} \frac{x_{\mathrm{A}}^{\prime \prime}}{x_{\mathrm{A}}^{\prime}}=\left(y_{\mathrm{D}}^{\prime \prime}-y_{\mathrm{D}}^{\prime}\right) x_{\mathrm{w}}^{\prime \prime}+y_{\mathrm{D}}^{\prime} \frac{x_{\mathrm{W}}^{\prime \prime}-x_{\mathrm{W}}^{\prime}}{1-x_{\mathrm{w}}^{\prime}}
$$

$$
x_{\mathrm{H}}^{\prime \prime}-x_{\mathrm{H}}^{\prime} \frac{x_{\mathrm{A}}^{\prime \prime}}{x_{\mathrm{A}}^{\prime}}=-\left(y_{\mathrm{D}}^{\prime \prime}-y_{\mathrm{D}}^{\prime}\right) x_{\mathrm{w}}^{\prime \prime}+\left(1-y_{\mathrm{D}}^{\prime}\right) \frac{x_{\mathrm{W}}^{\prime \prime}-x_{\mathrm{W}}^{\prime}}{1-x_{\mathrm{w}}^{\prime}}
$$

The variables, $z_{1}$ and $z_{2}$ in eqn. 6 or 7 may represent $y_{\mathrm{D}}$ and $x_{\mathrm{W}}$; correspondingly eqn. 7 yields two equations

$$
\begin{aligned}
& {\left[\frac{x_{\mathrm{w}}^{\prime \prime}-x_{\mathrm{w}}^{\prime}}{1-x_{\mathrm{w}}^{\prime}}\left(y_{\mathrm{D}}^{\prime}\left(\partial \mu_{\mathrm{D}}^{\prime} / \partial x_{\mathrm{W}}\right)_{T, y_{\mathrm{D}}}+\left(1-y_{\mathrm{D}}^{\prime}\right)\left(\partial \mu_{\mathrm{H}}^{\prime} / \partial x_{\mathrm{W}}\right)_{T, y_{\mathrm{D}}}\right)+x_{\mathrm{W}}^{\prime \prime}\left(y_{\mathrm{D}}^{\prime \prime}-y_{\mathrm{D}}^{\prime}\right)\left(\left(\partial \mu_{\mathrm{D}}^{\prime} / \partial x_{\mathrm{W}}\right)_{T, y_{\mathrm{D}}}\right.\right.} \\
& \left.\left.-\left(\partial \mu_{\mathrm{H}}^{\prime} / \partial x_{\mathrm{W}}\right)_{T, y_{\mathrm{D}}}\right)\right] \\
& \quad=\left(\mathrm{d} T / \mathrm{d} x_{\mathrm{W}}^{\prime}\right)_{\mathrm{eq}, y_{\mathrm{D}}^{\prime}}\left[x_{\mathrm{w}}^{\prime \prime}\left(y_{\mathrm{D}}^{\prime \prime}\left(S_{\mathrm{D}}^{\prime}-S_{\mathrm{D}}^{\prime \prime}\right)+\left(1-y_{\mathrm{D}}^{\prime \prime}\right)\left(S_{\mathrm{H}}^{\prime}-S_{\mathrm{H}}^{\prime \prime}\right)\right)+\left(1-x_{\mathrm{W}}^{\prime \prime}\right)\left(S_{\mathrm{A}}^{\prime}-S_{\mathrm{A}}^{\prime \prime}\right)\right]
\end{aligned}
$$

* When partial derivatives, as here, are supplied with dash, e.g. $\partial \mu^{\prime} / \partial x_{\mathrm{w}}$, it means $\left\langle\partial \mu / \partial x_{\mathrm{w}}\right\rangle T, y_{\mathrm{D}}$ at $x_{\mathrm{w}}=x_{\mathrm{w}}^{\prime}, y_{\mathrm{D}}=y_{\mathrm{D}}^{\prime}$ and $T=T_{\text {eq }}$ where $\left(x_{\mathrm{w}}^{\prime}, y_{\mathrm{D}}^{\prime}, T_{\text {eq }}\right)$ is a point on the solubility surface. 
and

$$
\begin{aligned}
& {\left[\frac{x_{\mathrm{W}}^{\prime \prime}-x_{\mathrm{w}}^{\prime}}{1-x_{\mathrm{W}}^{\prime}}\left(y_{\mathrm{D}}^{\prime}\left(\partial \mu_{\mathrm{D}}^{\prime} / \partial y_{\mathrm{D}}\right)_{T, x_{\mathrm{W}}}+\left(1-\ddot{y}_{\mathrm{D}}^{\prime}\right)\left(\partial \mu_{\mathrm{H}}^{\prime} / \partial y_{\mathrm{D}}\right)_{T, x_{\mathrm{W}}}\right)+x_{\mathrm{W}}^{\prime \prime}\left(y_{\mathrm{D}}^{\prime \prime}-y_{\mathrm{D}}^{\prime}\right)\left(\left(\partial \mu_{\mathrm{D}}^{\prime} / \partial y_{\mathrm{D}}\right)_{T, x_{\mathrm{W}}}\right.\right.} \\
& \left.\left.-\left(\partial \mu_{\mathrm{H}}^{\prime} / \partial y_{\mathrm{D}}\right)_{T, x_{\mathrm{W}}}\right)\right] \\
& \quad=\left(\mathrm{d} T / \mathrm{d} y_{\mathrm{D}}^{\prime}\right)_{\mathrm{eq}, x_{\mathrm{W}}}^{\prime}\left[x_{\mathrm{W}}^{\prime \prime}\left(y_{\mathrm{D}}^{\prime \prime}\left(S_{\mathrm{D}}^{\prime}-S_{\mathrm{D}}^{\prime \prime}\right)+\left(1-y_{\mathrm{D}}^{\prime \prime}\right)\left(S_{\mathrm{H}}^{\prime}-S_{\mathrm{H}}^{\prime \prime}\right)\right)+\left(1-x_{\mathrm{W}}^{\prime \prime}\right)\left(S_{\mathrm{A}}^{\prime}-S_{\mathrm{A}}^{\prime \prime}\right)\right]
\end{aligned}
$$

Introducing

$$
\left(\mathrm{d} T / \mathrm{d} y_{\mathrm{D}}^{\prime}\right)_{\mathrm{eq}, x_{\mathrm{w}}}^{\prime}=-\left(\mathrm{d} T / \mathrm{d} x_{\mathrm{w}}^{\prime}\right)_{\mathrm{eq}, y_{\mathrm{D}}}^{\prime}\left(\mathrm{d} x_{\mathrm{w}}^{\prime} / \mathrm{d} y_{\mathrm{D}}^{\prime}\right)_{\mathrm{eq}, T}
$$

a third equation involving $\left(\mathrm{d} x_{\mathrm{w}}^{\prime} / \mathrm{d} y_{\mathrm{D}}^{\prime}\right)_{\text {eq, } T}$ is obtained by combining eqns. 12 and 13:

$$
\begin{aligned}
& -\left[\frac{x_{\mathrm{W}}^{\prime \prime}-x_{\mathrm{W}}^{\prime}}{1-x_{\mathrm{W}}^{\prime}}\left(y_{\mathrm{D}}^{\prime}\left(\partial \mu_{\mathrm{D}}^{\prime} / \partial x_{\mathrm{W}}\right)_{T, y_{\mathrm{D}}}+\left(1-y_{\mathrm{D}}^{\prime}\right)\left(\partial \mu_{\mathrm{H} /}^{\prime} \partial x_{\mathrm{W}}\right)_{T, y_{\mathrm{D}}}\right)\right. \\
& \left.+x_{\mathrm{W}}^{\prime \prime}\left(y_{\mathrm{D}}^{\prime \prime}-y_{\mathrm{D}}^{\prime}\right)\left(\left(\partial \mu_{\mathrm{D}}^{\prime} / \partial x_{\mathrm{W}}\right)_{T, y_{\mathrm{D}}}-\left(\partial \mu_{\mathrm{H}}^{\prime} / \partial x_{\mathrm{W}}\right)_{T, y_{\mathrm{D}}}\right)\right]\left(\mathrm{d} x_{\mathrm{W}}^{\prime} / \mathrm{d} y_{\mathrm{D}}^{\prime}\right) \\
& =\left[\frac{x_{\mathrm{W}}^{\prime \prime}-x_{\mathrm{W}}^{\prime}}{1-x_{\mathrm{W}}^{\prime}}\left(y_{\mathrm{D}}^{\prime}\left(\partial \mu_{\mathrm{D}}^{\prime} / \partial y_{\mathrm{D}}\right)_{T, x_{\mathrm{W}}}+\left(1-y_{\mathrm{D}}^{\prime}\right)\left(\partial \mu_{\mathrm{H}}^{\prime} / \partial y_{\mathrm{D}}\right)_{T, x_{\mathrm{W}}}\right)\right. \\
& \left.+x_{\mathrm{W}}^{\prime \prime}\left(y_{\mathrm{D}}^{\prime \prime}-y_{\mathrm{D}}^{\prime}\right)\left(\left(\partial \mu_{\mathrm{D}}^{\prime} / \partial y_{\mathrm{D}}\right)_{T, x_{\mathrm{W}}}-\left(\partial \mu_{\mathrm{H}}^{\prime} / \partial y_{\mathrm{D}}\right)_{T, x_{\mathrm{W}}}\right)\right]
\end{aligned}
$$

$\Delta H$, the partial molar enthalpy of mixing, may be substituted for $S$, the partial molar entropy, in the following way:

$$
\begin{gathered}
\Delta H-T\left(S-S_{\mathrm{o}}\right)=\mu-\mu_{\mathrm{o}} \text { and } \mu^{\prime}-\mu_{\mathrm{o}}=\mu^{\prime \prime}-\mu_{\mathrm{o}} \text { so } \\
S_{\mathrm{D}}^{\prime}-S_{\mathrm{D}}^{\prime \prime}=S_{\mathrm{D}}^{\prime}-S_{\mathrm{Do}}-\left(S_{\mathrm{D}}^{\prime \prime}-S_{\mathrm{Do}}\right)=\left(\Delta H_{\mathrm{D}}^{\prime}-\Delta H_{\mathrm{D}}^{\prime \prime}\right) / T, \text { etc. }
\end{gathered}
$$

where subscript o refers to the pure state.

Eqn. 13 or 15 may form the basis for a calculation of $y_{\mathrm{D}}^{\prime \prime}-y_{\mathrm{D}}^{\prime}$ and thereby the isotope separation effect, $\left[y_{\mathrm{D}}^{\prime \prime}-y_{\mathrm{D}}^{\prime}\right] /\left[y_{\mathrm{D}}^{\prime}\left(1-y_{\mathrm{D}}^{\prime \prime}\right)\right]$ (while the term in eqn. 12 containing $\left(y_{\mathrm{D}}^{\prime \prime}-y_{\mathrm{D}}^{\prime}\right)$ is of second order only).

\section{Approximations}

2. 1. Basic considerations. Eqns. 12,13 and 15 are valid regardless of the number of chemical species, due to reactions between the three components, that may be present in the two phases as long, as full equilibrium has been reached. It should be noted, however, that the formulation of approximate chemical potential functions is simplified when information about the presence or non-presence of chemical species, can be introduced. In general three types of reactions leading to the formation of new chemical species should be considered:

Acta Chem. Scand. 16 (1962) No. 7 
1) Equilibrium isotope exchange with the organic component according to the following reaction scheme

$$
\frac{1}{2} \mathrm{D}_{2} \mathrm{O}+\mathrm{HB} \rightleftharpoons \frac{1}{2} \mathrm{H}_{2} \mathrm{O}+\mathrm{DB}
$$

where $\mathrm{HB}$ is added organic component and $\mathrm{DB}$ is the same deuterated. TEA exchanges slowly, if at all, with $\mathrm{D}_{2} \mathrm{O}$. The following theoretical considerations are valid only when this kind of reaction can be neglected.

2) Reaction with the organic component of the following type:

$$
\mathrm{H}_{2} \mathrm{O}+\mathrm{TEA} \rightleftharpoons \mathrm{TEAH}^{+}+\mathrm{OH}^{-} \text {. }
$$

Such reaction products may be present in the $\mathrm{D}_{2} \mathrm{O}-\mathrm{H}_{2} \mathrm{O}-\mathrm{TEA}$ system. No accurate information is available and is, in fact, thought to be unnecessary for the following description of the system (where the parameters $\left[\mathrm{D}_{2} \mathrm{O}\right]$ and $\left[\mathrm{H}_{2} \mathrm{O}\right]$ include both ions and neutral molecules, and any isotope effects in the reactions are disregarded.) See below (p. 1742) for further comments.

$$
\mathrm{D}_{2} \mathrm{O}+\mathrm{H}_{2} \mathrm{O} \rightleftharpoons 2 \mathrm{HDO}
$$

This reaction takes place to a degree determined by

$$
\frac{f_{\mathrm{HDO}}^{2}[\mathrm{HDO}]^{2}}{f_{\mathrm{H}} f_{\mathrm{D}}\left[\mathrm{H}_{2} \mathrm{O}\right]\left[\mathrm{D}_{2} \mathrm{O}\right]}=K^{*} \text { where } K^{*} \simeq 4
$$

and $f_{\mathrm{D}}, f_{\mathrm{H}}$ and $f_{\mathrm{HDO}}$ are the activity coefficients of $\mathrm{D}_{2} \mathrm{O}, \mathrm{H}_{2} \mathrm{O}$, and HDO, respectively.

By taking into account that $K \equiv \frac{1}{4} \cdot \frac{f_{\mathrm{H}} f_{\mathrm{D}}}{f_{\mathrm{HDO}}^{2}} K^{*}$ is near one (see below) and introducing the two conditions that

and

$$
\begin{aligned}
& {\left[\mathrm{D}_{2} \mathrm{O}\right]+\left[\mathrm{H}_{2} \mathrm{O}\right]+[\mathrm{HDO}]=x_{\mathrm{W}}} \\
& {\left[\mathrm{D}_{2} \mathrm{O}\right]+\frac{1}{2}[\mathrm{HDO}]=y_{\mathrm{D}} x_{\mathrm{W}}}
\end{aligned}
$$

one obtains the following three equations

$$
\begin{aligned}
& {\left[\mathrm{D}_{2} \mathrm{O}\right]=(1+a) y_{\mathrm{D}}^{2} x_{\mathrm{W}}} \\
& {\left[\mathrm{H}_{2} \mathrm{O}\right]=(\mathbf{1}+b)\left(1-y_{\mathrm{D}}\right)^{2} x_{\mathrm{W}}} \\
& {[\mathrm{HDO}]=\mathbf{2}(\mathbf{1}+c) y_{\mathrm{D}}\left(\mathbf{1}-y_{\mathrm{D}}\right) x_{\mathrm{W}}}
\end{aligned}
$$

Here $a, b$ and $c$ are small compared to one, so the approximation may be made that

$$
K=\frac{(1+c)^{2}}{(1+a)(1+b)} \simeq 1+2 c-a-b
$$

then $a, b$, and $c$ can be expressed in the following way

$$
\begin{aligned}
a & =-(K-1)\left(1-y_{\mathrm{D}}\right)^{2} \\
b & =-(K-1) y_{\mathrm{D}}^{2} \\
c & =(K-1) y_{\mathrm{D}}\left(1-y_{\mathrm{D}}\right)
\end{aligned}
$$

In pure $\mathrm{D}_{2} \mathrm{O}-\mathrm{H}_{2} \mathrm{O}$ mixtures $K=0.83$ (Ref. ${ }^{3}$ ) and the same must be approximately the case in the TEA-water mixture, since $f_{\mathrm{DHO}}$ must be near $\sqrt{f_{\mathrm{D}} f_{\mathrm{H}}}$.

2.2. First approximation. The change in chemical potential with composition may be written 


$$
\left(\frac{\partial \mu_{\mathrm{D}}}{\partial z_{1}}\right)_{T, z_{z}}=R T\left(\frac{\partial \ln \left[\mathrm{D}_{2} \mathrm{O}\right]}{\partial z_{1}}\right)_{T, z_{2}}+R T\left(\frac{\partial \ln f_{\mathrm{D}}}{\partial z_{1}}\right)_{T, z_{3}}
$$

and similarly for $\mu_{\mathrm{H}}$ and $\mu_{\mathrm{A}}$. The three partial derivatives, $\left(\partial \ln f / \partial y_{\mathrm{D}}\right)_{T, x_{\mathrm{W}}}$, result from changes in the medium (triethylamine phase) when $\mathrm{D}_{2} \mathrm{O}$ is substituted for $\mathrm{H}_{2} \mathrm{O}$ at constant water content $\left(x_{\mathrm{w}}\right)$; while the three partial derivatives, $\left(\partial \ln f / \partial x_{\mathrm{W}}\right)_{T, y_{\mathrm{D}}}$ result from changes in the medium when the water content is increased at constant $y_{\mathrm{D}}$, the isotopic composition. $\left|\partial \ln f / \partial y_{\mathrm{D}}\right|$ must obviously be considerably smaller than the corresponding $|\partial \ln f| \partial x_{\mathrm{w}} \mid$, so it would seem to be a justifiable first approximation to assume that

$$
\partial \ln f / \partial y_{\mathrm{D}}=0
$$

This has the following implications:

1) $K$ (eqn. 23) will be independent of $y_{\mathrm{D}}$.

2) (eqn. 22): $\left(\frac{\partial \mu_{\mathrm{D}}}{\partial y_{\mathrm{D}}}\right)_{T, x_{\mathrm{W}}}=R T\left(\frac{\partial \ln \left[\mathrm{D}_{2} \mathrm{O}\right]}{\partial y_{\mathrm{D}}}\right)_{T, x_{\mathrm{W}}}=\frac{2 R T}{y_{\mathrm{D}}}+R T\left(\frac{\partial \ln (1+a)}{\partial y_{\mathrm{D}}}\right)_{T, x_{\mathrm{W}}}$

$$
\left(\frac{\partial \mu_{\mathrm{H}}}{\partial y_{\mathrm{D}}}\right)_{T, x_{\mathrm{W}}}=R T\left(\frac{\partial \ln \left[\mathrm{H}_{2} \mathrm{O}\right]}{\partial y_{\mathrm{D}}}\right)_{T, x_{\mathrm{W}}}=-\frac{2 R T}{1-y_{\mathrm{D}}}+R T\left(\frac{\partial \ln (1+b)}{\partial y_{\mathrm{D}}}\right)_{T, x_{\mathrm{W}}}
$$

Here $\frac{\partial \ln (1+a)}{\partial y_{\mathrm{D}}}=(K-1) 2\left(1-y_{\mathrm{D}}\right)$ and $\frac{\partial \ln (1+b)}{\partial y_{\mathrm{D}}}=-(K-1) 2 y_{\mathrm{D}}$

These two derivatives are fairly small correction terms, so

$$
\begin{gathered}
y_{\mathrm{D}} \frac{\partial \mu_{\mathrm{D}}}{\partial y_{\mathrm{D}}}+\left(1-y_{\mathrm{D}}\right) \frac{\partial \mu_{\mathrm{D}}}{\partial y_{\mathrm{D}}}=0 \text { and } \\
\frac{\partial \mu_{\mathrm{D}}}{\partial y_{\mathrm{D}}}-\frac{\partial \mu_{\mathrm{H}}}{\partial y_{\mathrm{D}}}=\frac{2 R T}{y_{\mathrm{D}}}+\frac{2 R T}{1-y_{\mathrm{D}}}+2 R T(K-1) \simeq \frac{2 R T}{y_{\mathrm{D}}\left(1-y_{\mathrm{D}}\right)}
\end{gathered}
$$

Neglecting $K-1$ probably causes only a $5 \%$ error at maximum, at $y_{\mathrm{D}}=\frac{1}{2}$.

3) From $\partial \ln f_{\mathrm{A}} / \partial y_{\mathrm{D}}=0$ at any $x_{\mathrm{w}}$ and the Gibbs-Duhem equation applied to pure $\mathrm{H}_{2} \mathrm{O}-\mathrm{TEA}$ and $\mathrm{D}_{2} \mathrm{O}-\mathrm{TEA}$ mixtures as follows:

and

$$
x_{\mathrm{w}} \frac{\partial \ln f_{\mathrm{Hh}}}{\partial x_{\mathrm{W}}}+\left(1-x_{\mathrm{W}}\right) \frac{\partial \ln f_{\mathrm{Ah}}}{\partial x_{\mathrm{w}}}=0
$$

it follows that

$$
x_{\mathrm{W}} \frac{\partial \ln f_{\mathrm{Dd}}}{\partial x_{\mathrm{W}}}+\left(1-x_{\mathrm{w}}\right) \frac{\partial \ln f_{\mathrm{Ad}}}{\partial x_{\mathrm{w}}}=0
$$

and

$$
\frac{\partial \mu_{\mathrm{D}}}{\partial x_{\mathrm{w}}}=\frac{\partial \mu_{\mathrm{H}}}{\partial x_{\mathrm{w}}} \equiv \frac{\partial \mu_{\mathrm{w}}}{\partial x_{\mathrm{w}}} \text { at any } y_{\mathrm{D}}
$$

$$
y_{\mathrm{D}} \frac{\partial \mu_{\mathrm{D}}}{\partial x_{\mathrm{w}}}+\left(1-y_{\mathrm{D}}\right) \frac{\partial \mu_{\mathrm{H}}}{\partial x_{\mathrm{w}}}=\frac{\partial \mu_{\mathrm{w}}}{\partial x_{\mathrm{w}}}
$$

Acta Chem. Scand. 16 (1962) No. 7 


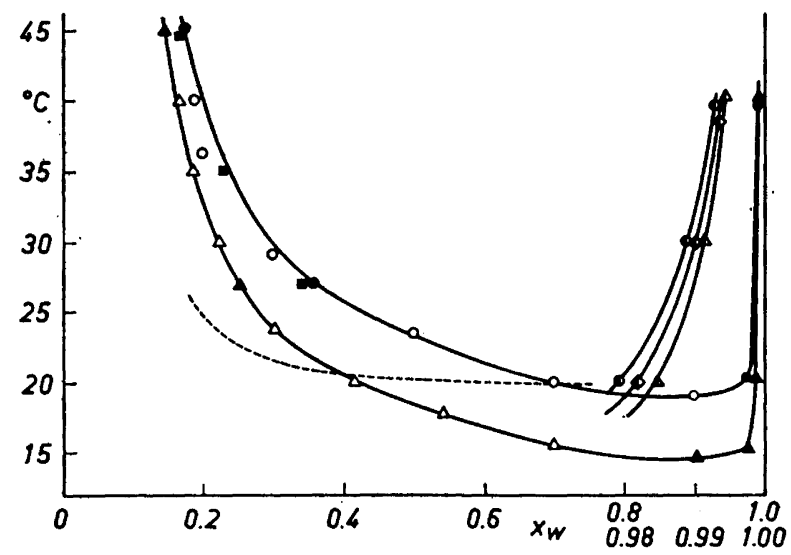

Fig. 2. Solubility curves for mixtures of triethylamine, water and heavy water. $x_{\mathrm{w}}$ is mole fraction of water $\left(\mathrm{D}_{2} \mathrm{O}+\mathrm{H}_{2} \mathrm{O}\right)$. The water phase data have been determined by titration.

\begin{tabular}{|c|c|c|c|}
\hline \multirow{2}{*}{$y_{\mathrm{D}}$} & \multicolumn{2}{|c|}{ Own data } & \multirow{2}{*}{$\begin{array}{c}\text { Hahn and Street' } \\
\text { data }^{2} \\
\text { (main } x_{w^{-}} \text {scale) }\end{array}$} \\
\hline & $\underset{x_{\mathrm{w}} \text {-scale }}{\operatorname{main}}$ & $\begin{array}{l}\text { enlarged } \\
x_{\mathrm{w}} \text {-scale }\end{array}$ & \\
\hline $\begin{array}{l}0 \\
0.20\end{array}$ & 음 & $\boldsymbol{D}$ & 0 \\
\hline 0.50 & & $\Delta$ & \\
\hline 1.00 & $\boldsymbol{\Delta}$ & $\Delta$ & $\triangle$ \\
\hline
\end{tabular}

The dashed curve corresponds to the data of Rothmund ${ }^{9}\left(y_{\mathrm{D}}=0\right)$.

4) With sufficient accuracy it may be assumed that

$$
\Delta \mathrm{H}_{\mathrm{D}}=\Delta \mathrm{H}_{\mathrm{H}} \equiv \Delta H_{\mathrm{W}}
$$

These approximations do not imply that there is no difference between $f_{\mathrm{D}}$ and $f_{\mathrm{H}}$ - the activity coefficients of $\mathrm{D}_{2} \mathrm{O}$ and $\mathrm{H}_{2} \mathrm{O}$ compared at equal $x_{\mathrm{W}}$ but do imply that the activities or vapour pressures of $\mathrm{D}_{2} \mathrm{O}$ and $\mathrm{H}_{2} \mathrm{O}$ at any $x_{\mathrm{w}}$ are proportional to $\left[\mathrm{D}_{2} \mathrm{O}\right]$ and $\left[\mathrm{H}_{2} \mathrm{O}\right]$, respectively, and that the activity or vapour pressure of TEA at any $x_{\mathrm{w}}$ is independent of $y_{\mathrm{D}}$. If the latter statement is true for the triethylamine phase it cannot be simultaneously true for the water phase, since the solubility curves (Fig. 2) do not intersect.

2.3. Second approximations. The approximate potential functions developed above give simple and useful equations when applied to the fundamental equilibrium conditions (eqns. 12,13 and 15); it is, however, of interest to estimate the magnitude of the error introduced by neglecting the derivatives $\partial \ln f / \partial y_{\mathrm{D}}$. The data available make in fact this possible if the assumption is made that all three derivatives are constants in the interval $0 \leqq y_{\mathrm{D}} \leqq 1$. This immediately necessitates (the Gibbs-Duhem eqn. 5) that

$$
\frac{\partial \ln f_{\mathrm{D}}}{\partial y_{\mathrm{D}}}=\frac{\partial \ln f_{\mathrm{H}}}{\partial y_{\mathrm{D}}}\left(=-\frac{1-x_{\mathrm{W}}}{x_{\mathrm{w}}} \frac{\partial \ln f_{\mathrm{A}}}{\partial y_{\mathrm{D}}}\right) \equiv M
$$

Acta Chem. Scand. 16 (1962) No. 7 
Therefore

$$
\ln f_{\mathrm{D}}-\ln f_{\mathrm{Dd}}=-M\left(1-y_{\mathrm{D}}\right)
$$

and

$$
\begin{gathered}
\ln f_{\mathrm{H}}-\ln f_{\mathrm{Hh}}=M y_{\mathrm{D}} \\
\text { so }\left(\frac{\partial \ln f_{\mathrm{D}}}{\partial x_{\mathrm{W}}}\right)_{T, y_{\mathrm{D}}}=\left(\frac{\partial \ln f_{\mathrm{Dd}}}{\partial x_{\mathrm{w}}}\right)_{T}-\left(1-y_{\mathrm{D}}\right)\left(\frac{\partial M}{\partial x_{\mathrm{w}}}\right)_{T} \\
\quad \text { and }\left(\frac{\partial \ln f_{\mathrm{H}}}{\partial x_{\mathrm{W}}}\right)_{T, y_{\mathrm{D}}}=\left(\frac{\partial \ln f_{\mathrm{Hh}}}{\partial x_{\mathrm{W}}}\right)_{T}+y_{\mathrm{D}}\left(\frac{\partial M}{\partial x_{\mathrm{w}}}\right)_{T}
\end{gathered}
$$

where $d$ and $h$ refers to pure $\mathrm{D}_{2} \mathrm{O}$ - and $\mathrm{H}_{2} \mathrm{O}$-triethylamine systems, respectively. Differentiating eqn. 34 gives

$$
\frac{\partial M}{\partial x_{\mathrm{w}}}=-\frac{1-x_{\mathrm{w}}}{x_{\mathrm{w}}} \frac{\partial}{\partial x_{\mathrm{w}}}\left(\frac{\partial \ln f_{\mathrm{A}}}{\partial y_{\mathrm{D}}}\right)+\frac{1}{x_{\mathrm{w}}^{2}} \frac{\partial \ln f_{\mathrm{A}}}{\partial y_{\mathrm{D}}}
$$

The following relations may now be derived:

1) Eqn. 34, with the resonable assumption that also $\partial \ln f_{\mathrm{DHO}} / \partial y_{\mathrm{D}}=M$, shows that $K$ of eqn. 23 under these conditions also becomes independent of $y_{\mathrm{D}}$.

2) From eqns. 34 (cf. eqns. 28 and 29) the following results are obtained:

$$
\begin{aligned}
y_{\mathrm{D}} \frac{\partial \mu_{\mathrm{D}}}{\partial y_{\mathrm{D}}}+\left(1-y_{\mathrm{D}}\right) \frac{\partial \mu_{\mathrm{H}}}{\partial y_{\mathrm{D}}}=R T M & =-R T \frac{1-x_{\mathrm{w}}}{x_{\mathrm{W}}} \frac{\partial \ln f_{\mathrm{A}}}{\partial y_{\mathrm{D}}} \\
& =-R T \frac{1-x_{\mathrm{W}}}{x_{\mathrm{w}}}\left(\ln \frac{f_{\mathrm{Ad}}}{f_{\mathrm{Ah}}}\right)_{x_{\mathrm{w}}}
\end{aligned}
$$

and $\quad \frac{\partial \mu_{\mathrm{D}}}{\partial y_{\mathrm{D}}}-\frac{\partial \mu_{\mathrm{H}}}{\partial y_{\mathrm{D}}} \simeq \frac{2 R T}{y_{\mathrm{D}}\left(1-y_{\mathrm{D}}\right)}$

3) From eqns. 36 and 37 it follows that

$$
\frac{\partial \ln f_{\mathrm{D}}}{\partial x_{\mathrm{W}}}-\frac{\partial \ln f_{\mathrm{H}}}{\partial x_{\mathrm{w}}}=\frac{\partial \ln \left(f_{\mathrm{Dd}} / f_{\mathrm{Hh}}\right)}{\partial x_{\mathrm{w}}}+\frac{1-x_{\mathrm{W}}}{x_{\mathrm{w}}} \frac{\partial}{\partial x_{\mathrm{w}}}\left(\frac{\partial \ln f_{\mathrm{A}}}{\partial y_{\mathrm{D}}}\right)-\frac{1}{x_{\mathrm{w}}^{2}} \frac{\partial \ln f_{\mathrm{A}}}{\partial y_{\mathrm{D}}}
$$

and introducing eqn. 30 gives

Eqn. 36 yields

$$
\frac{\partial \mu_{\mathrm{D}}}{\partial x_{\mathrm{w}}}-\frac{\partial \mu_{\mathrm{H}}}{\partial x_{\mathrm{w}}}=-\frac{R T}{x_{\mathrm{w}}^{2}}\left(\ln \frac{f_{\mathrm{Ad}}}{f_{\mathrm{Ah}}}\right)_{x_{\mathrm{w}}}
$$

$$
y_{\mathrm{D}} \frac{\partial \mu_{\mathrm{D}}}{\partial x_{\mathrm{W}}}+\left(1-y_{\mathrm{D}}\right) \frac{\partial \mu_{\mathrm{H}}}{\partial x_{\mathrm{W}}}=y_{\mathrm{D}} \frac{\partial \mu_{\mathrm{Dd}}}{\partial x_{\mathrm{W}}}+\left(1-y_{\mathrm{D}}\right) \frac{\partial \mu_{\mathrm{Hh}}}{\partial x_{\mathrm{w}}}
$$
of

4) Differentiation of eqn. 34 with respect to temperature and introduction

$$
\frac{\partial \ln f}{\partial T}=-\frac{\Delta H}{R T^{2}}
$$

Acta Chem. Scand. 16 (1962) No. : 
(where $\Delta H$ is the partial molar enthalpy of mixing) gives

So

$$
-\frac{\partial \Delta H_{\mathrm{D}}}{\partial y_{\mathrm{D}}}=-\frac{\partial \Delta H_{\mathrm{H}}}{\partial y_{\mathrm{D}}}=\frac{1-x_{\mathrm{W}}}{x_{\mathrm{W}}} \frac{\partial \Delta H_{\mathrm{A}}}{\partial y_{\mathrm{D}}}=R T^{2} \frac{\partial M}{\partial T}
$$

So $\quad-\Delta H_{\mathrm{D}}+\Delta H_{\mathrm{Dd}}=-R T^{2} \frac{\partial M}{\partial T}\left(1-y_{\mathrm{D}}\right)$

and $-\Delta H_{\mathrm{H}}+H_{\mathrm{Hh}}=R T^{2} \frac{\partial M}{\partial T} y_{\mathrm{D}}$

By subtraction $\left(\Delta H_{\mathrm{D}}-\Delta H_{\mathrm{H}}\right)-\left(\Delta H_{\mathrm{Dd}}-\Delta H_{\mathrm{Hh}}\right)=R T^{2} \frac{\partial M}{\partial T}$

while $\quad y_{\mathrm{D}} \Delta H_{\mathrm{D}}+\left(1-y_{\mathrm{D}}\right) \Delta H_{\mathrm{H}}=y_{\mathrm{D}} \Delta H_{\mathrm{Dd}}+\left(1-y_{\mathrm{D}}\right) \Delta H_{\mathrm{Hh}}$

The preceding systems of equations are based on the assumption that ideal conditions or conditions approaching the ideal are maintained when, in TEAwater mixtures, $\mathrm{D}_{2} \mathrm{O}$ is interchanged with $\mathrm{H}_{2} \mathrm{O}$, the total amount of water $\left(x_{\mathrm{w}}\right)$ being held constant. It is, however, possible, as mentioned earlier, that reactions of the following type take place: TEA $+\mathrm{H}_{2} \mathrm{O} \rightleftharpoons \mathrm{TEAH}^{+}+\mathrm{OH}^{-}$. The extent to which TEA reacts with $\mathrm{H}_{2} \mathrm{O}$ is probably not exactly the same as with $\mathrm{D}_{2} \mathrm{O}$. The ratio of the amounts of the two types of chemical species could have marked influence on the medium, so one could expect that a different parameter in place of $x_{\mathrm{w}}$ might be required. Infrared tests for the presence of $\mathrm{NH}^{+}$- and $\mathrm{ND}^{+}$-bonds showed that not more and probably much less than $10 \%$ of the water in the TEA-phase is in the ionized form. An approximate calculation shows that the presence of ions in that concentration probably has so little effect that it is taken into account in a satisfactory manner by the introduction of the second set of approximations (eqn. 34). A comparatively successful determination of $\ln \left(f_{\mathrm{Ad}} / f_{\mathrm{Ah}}\right)$ which is discussed below points in the same direction.

3. Application of the approximations to the equilibrium equations.

3.1. First approximations. Introducing the simple first approximations represented by eqns. 28, 29, 31, and 32 into eqns. 12, 13, and 15 gives

$$
\begin{gathered}
\left(\frac{\partial \mu_{\mathrm{W}}^{\prime}}{\partial x_{\mathrm{W}}}\right)_{T, y_{\mathrm{D}}}\left(\frac{\mathrm{d} x_{\mathrm{W}}^{\prime}}{\mathrm{d} T}\right)_{\mathrm{eq}, y_{\mathrm{D}}^{\prime}}=\frac{\Delta H_{\mathrm{W}}^{\prime}}{T} \\
2 R T \frac{y_{\mathrm{D}}^{\prime \prime}-y_{\mathrm{D}}^{\prime}}{y_{\mathrm{D}}^{\prime}\left(1-y_{\mathrm{D}}^{\prime}\right)}\left(\frac{\mathrm{d} y_{\mathrm{D}}^{\prime}}{\mathrm{d} T}\right)_{\mathrm{eq}, x_{\mathrm{W}}^{\prime}}=\frac{\Delta H_{\mathrm{W}}^{\prime}}{T} \\
\left(\frac{\partial \mu_{\mathrm{W}}^{\prime}}{\partial x_{\mathrm{W}}}\right)_{T, y_{\mathrm{D}}}\left(\frac{\mathrm{d} x_{\mathrm{W}}^{\prime}}{\mathrm{d} y_{\mathrm{D}}^{\prime}}\right)_{\mathrm{eq}, T}=-2 R T \frac{y_{\mathrm{D}}^{\prime \prime}-y_{\mathrm{D}}^{\prime}}{y_{\mathrm{D}}^{\prime}\left(1-y_{\mathrm{D}}^{\prime}\right)}
\end{gathered}
$$

where $x_{\mathrm{W}}^{\prime \prime}$ has been set equal to one, $\Delta H_{\mathrm{W}}^{\prime \prime}$ equal to zero and where $\left(1-x_{\mathrm{W}}^{\prime \prime}\right)$ $\left(\Delta H_{\mathrm{A}}^{\prime}-\Delta H_{\mathrm{A}}^{\prime \prime}\right)<\left\langle\Delta H_{\mathrm{W}}^{\prime}\right.$ is neglected (see Fig. 2).

The isotope separation effect, $\left[y_{\mathrm{D}}^{\prime \prime}\left(1-y_{\mathrm{D}}^{\prime}\right) / y_{\mathrm{D}}^{\prime}\left(1-y_{\mathrm{D}}^{\prime \prime}\right)\right]-1$, for all practical purposes equal to $\left(y_{\mathrm{D}}^{\prime \prime}-y_{\mathrm{D}}^{\prime}\right) / y_{\mathrm{D}}^{\prime}\left(1-y_{\mathrm{D}}^{\prime}\right)$, may now be calculated from either eqn. 48 or 49 using available data for the partial vapour pressure of $\mathrm{H}_{2} \mathrm{O}$ in 
TEA $-\mathrm{H}_{2} \mathrm{O}$ mixtures and available determinations of the heat of mixing of TEA and $\mathrm{H}_{2} \mathrm{O}$, together with the solubility curves.

Eqn. 49 is analogous to the Duhem-Margules equation (in one of its forms) for a binary liquid mixture (e.g. $\mathrm{D}_{2} \mathrm{O}$ and $\mathrm{H}_{2} \mathrm{O}$ ) in equilibrium with its vapour: in fact, substituting in eqns. 3 to $5 x_{\mathrm{A}} \mathrm{d} \mu_{\mathrm{A}}$ for $v \cdot \mathrm{d} p$ (where $v$ is the molar volume of the gasous mixture and $p$ the total pressure) and choosing as independent variables $y_{\mathrm{D}}$ (the composition of the vapour) (or $x_{\mathrm{D}}$, since $x_{\mathrm{W}}=1$ ) and $p$, one arrives at three equations analogous to eqns. 47 to 49 .

$$
\begin{gathered}
R T\left(\frac{\mathrm{d} \ln p}{\mathrm{~d} T}\right)_{\mathrm{eq}, y}=y_{\mathrm{D}}^{\prime \prime}\left(S_{\mathrm{D}}^{\prime}-S_{\mathrm{D}}^{\prime \prime}\right)+\left(1-y_{\mathrm{D}}^{\prime \prime}\right)\left(S_{\mathrm{H}}^{\prime}-S_{\mathrm{H}}^{\prime \prime}\right) \\
2 R T \frac{y_{\mathrm{D}}^{\prime \prime}-y_{\mathrm{D}}^{\prime}}{y_{\mathrm{D}}^{\prime}\left(1-y_{\mathrm{D}}^{\prime}\right)}\left(\frac{\mathrm{d} y_{\mathrm{D}}^{\prime}}{\mathrm{d} T}\right)_{\mathrm{eq}, p}=y_{\mathrm{D}}^{\prime \prime}\left(S_{\mathrm{D}}^{\prime}-S_{\mathrm{D}}^{\prime \prime}\right)+\left(1-y_{\mathrm{D}}^{\prime \prime}\right)\left(S_{\mathrm{H}}^{\prime}-S_{\mathrm{H}}^{\prime \prime}\right)
\end{gathered}
$$

and the Duhem-Margules equation:

$$
R T\left(\frac{\mathrm{d} \ln p}{\mathrm{~d} y_{\mathrm{D}}^{\prime}}\right)_{\mathrm{eq}, T}=-2 R T \frac{y_{\mathrm{D}}^{\prime \prime}-y_{\mathrm{D}}^{\prime}}{y_{\mathrm{D}}^{\prime}\left(1-y_{\mathrm{D}}^{\prime}\right)}
$$

Eqn. 50 is the equation for the vapour pressure curve.

From eqn. 51 the separation effect may be calculated when the heat of evaporation (and, if necessary, of solution) and the boiling point as a function of vapour composition are known.

Eqn. 52 relates the relative volatility of the pure components to the separation effects. If, in eqn. $49, \partial \mu_{\mathrm{W}}^{\prime} / \partial x_{\mathrm{w}}$ is set equal to $R T / x_{\mathrm{w}}^{\prime}$, eqns. 49 and 52 become identical in form. It will be shown below that this assumption is not at all permissible; however, eqn. 49, simplified in this manner, is of interest because it may have formed the basis for optimistic estimations of the isotope separation potentialities of this system and other similar systems.

3.2. Second approximations. Introducing the second approximation represented by eqns. $38,39,40$, and 41 into eqns. 12, 13, and 15 gives: (eqn. 12)

$$
\frac{x_{\mathrm{W}}^{\prime \prime}-x_{\mathrm{W}}^{\prime}}{1-x_{\mathrm{W}}^{\prime}}\left(y_{\mathrm{D}}^{\prime} \frac{\partial \mu_{\mathrm{Dd}}}{\partial x_{\mathrm{w}}}+\left(1-y_{\mathrm{D}}^{\prime}\right) \frac{\partial \mu_{\mathrm{Hh}}}{\partial x_{\mathrm{W}}}\right)-x_{\mathrm{W}}^{\prime \prime} \frac{\left(y_{\mathrm{D}}^{\prime \prime}-y_{\mathrm{D}}^{\prime}\right)}{x_{\mathrm{w}}^{\prime}{ }^{2}} R T\left(\ln \frac{f_{\mathrm{Ad}}}{f_{\mathrm{Ah}}}\right)_{x^{\prime} \mathrm{w}}=
$$

$\left(\frac{\mathrm{d} T}{\mathrm{~d} x_{\mathrm{W}}^{\prime}}\right)_{\mathrm{eq}, y_{\mathrm{D}}^{\prime}}\left[x_{\mathrm{w}}\left(y_{\mathrm{D}}^{\prime \prime} \frac{\Delta H_{\mathrm{D}}^{\prime}-\Delta H_{\mathrm{D}}^{\prime \prime}}{T}+\left(1-y_{\mathrm{D}}^{\prime \prime}\right) \frac{\Delta H_{\mathrm{H}}^{\prime}-\Delta H_{\mathrm{H}}^{\prime \prime}}{T}\right)+\left(1-x_{\mathrm{W}}^{\prime \prime}\right) \frac{\Delta H_{\mathrm{A}}^{\prime}-\Delta H_{\mathrm{A}}^{\prime \prime}}{T}\right]$

(eqn. 13)

$$
-\frac{x_{\mathrm{W}}^{\prime \prime}-x_{\mathrm{W}}^{\prime}}{x_{\mathrm{W}}^{\prime}}\left(\ln \frac{f_{\mathrm{Ad}}}{f_{\mathrm{Ah}}}\right)_{x^{\prime} \mathrm{W}} R T+2 R T \frac{x_{\mathrm{W}}^{\prime \prime}\left(y_{\mathrm{D}}^{\prime \prime}-y_{\mathrm{D}}^{\prime}\right)}{y_{\mathrm{D}}^{\prime}\left(1-y_{\mathrm{D}}^{\prime}\right)}=
$$

$\left(\frac{\mathrm{d} T}{\mathrm{~d} y_{\mathrm{D}}^{\prime}}\right)_{\mathrm{eq}, x^{\prime}{ }_{\mathrm{W}}}\left[x_{\mathrm{W}}^{\prime \prime}\left(y_{\mathrm{D}}^{\prime \prime} \frac{\Delta H_{\mathrm{D}}^{\prime}-\Delta H_{\mathrm{D}}^{\prime \prime}}{T}+\left(1-y_{\mathrm{D}}^{\prime \prime}\right) \frac{\Delta H_{\mathrm{H}}^{\prime}-\Delta H_{\mathrm{H}}^{\prime \prime}}{T}\right)+\left(1-x_{\mathrm{W}}^{\prime \prime}\right) \frac{\Delta H_{\mathrm{A}}^{\prime}-\Delta H_{\mathrm{A}}^{\prime \prime}}{T}\right]$

Acta Chem. Scand. 16 (1962) No. 7 
(eqn. 15)

$$
\begin{aligned}
& {\left[\frac{x_{\mathrm{w}}^{\prime \prime}-x_{\mathrm{w}}^{\prime}}{1-x_{\mathrm{w}}^{\prime}}\left(y_{\mathrm{D}}^{\prime} \frac{\partial \mu_{\mathrm{Dd}}}{\partial x_{\mathrm{w}}}+\left(1-y_{\mathrm{D}}^{\prime}\right) \frac{\partial \mu_{\mathrm{Hh}}}{\partial x_{\mathrm{w}}}\right)-\frac{x_{\mathrm{w}}^{\prime \prime}\left(y_{\mathrm{D}}^{\prime \prime}-y_{\mathrm{D}}^{\prime}\right)}{x_{\mathrm{w}}^{\prime 2}} R T\left(\ln \frac{f_{\mathrm{Ad}}}{f_{\mathrm{Ah}}}\right)_{x_{\mathrm{w}}^{\prime}}\right] \times} \\
& \quad \times\left(\frac{\mathrm{d} x_{\mathrm{W}}^{\prime}}{\mathrm{d} y_{\mathrm{D}}^{\prime}}\right)_{\mathrm{eq}, T}=\frac{x_{\mathrm{w}}^{\prime \prime}-x_{\mathrm{w}}^{\prime}}{x_{\mathrm{W}}^{\prime}}\left(\ln \frac{f_{\mathrm{Ad}}}{f_{\mathrm{Ah}}}\right)_{x_{\mathrm{w}}} R T-2 R T \frac{x_{\mathrm{w}}^{\prime \prime}\left(y_{\mathrm{D}}^{\prime \prime}-y_{\mathrm{D}}^{\prime}\right)}{y_{\mathrm{D}}^{\prime}\left(1-y_{\mathrm{D}}^{\prime}\right)}
\end{aligned}
$$

(It should be noted that $\left(\partial \mu_{\mathrm{Dd}} / \partial x_{\mathrm{W}}\right)_{x_{\mathrm{W}}=x^{\prime}{ }_{\mathrm{W}}}$ and $\left(\ln f_{\mathrm{Ad}}\right)_{x^{\prime} \mathrm{W}}$ apply to the unstable region, because $x_{\mathrm{W}}^{\prime}>x_{\mathrm{W}, d}^{\prime}$ (the solubility of $\mathrm{D}_{2} \mathrm{O}$ ).)

The terms that distinguish eqns. 53-55 from eqns. 47-49 contain $\ln \left(f_{\mathrm{Ad}} / f_{\mathrm{Ah}}\right)$. When therefore an estimate of this quantity has been made, it will be possible to calculate the separation effect by eqn. 54 or 55 . The difference between $\partial \mu_{\mathrm{Dd}} / \partial x_{\mathrm{w}}$ and $\partial \mu_{\mathrm{Hh}} / \partial x_{\mathrm{W}}$ and between $\Delta H_{\mathrm{D}}$ and $\Delta H_{\mathrm{H}}$ may be neglected, at least at low $y_{\mathrm{D}}^{\prime}$.

3.3. $\ln \left(f_{\mathrm{Ad}} / f_{\mathrm{Ah}}\right)$ could be easily calculated, if the vapour pressure curve of pure $\mathrm{D}_{2} \mathrm{O}$-TEA mixtures were known. Failing this the same information may in principle be obtained from the solubility curves (Kohler ${ }^{4}$ ); however the accuracy of this approach is limited so that the following treatment may furnish only the order of magnitude of $\ln \left(f_{\mathrm{Ad}} / f_{\mathrm{Ah}}\right)$.

3.3.1. First an expression for $\left(\ln \left(f_{\mathrm{Dd}} / f_{\mathrm{Hh}}\right)\right)_{x_{\mathrm{w}}}$ is to be found:

Corresponding to eqn. 1 the following two equations may be written, inserting eqn. 22, for $\mu_{\mathrm{D}}$

$$
\begin{aligned}
& \text { at } T=T_{\mathrm{D}}, x_{\mathrm{W}}=x_{\mathrm{w}}^{\prime} \text { and } y_{\mathrm{D}}^{\prime}=1 \text { : } \\
& \ln x_{\mathrm{W}}^{\prime}+\ln f_{\mathrm{Dd}}^{\prime}=\ln x_{\mathrm{W}, \mathrm{D}}^{\prime \prime}+\ln f_{\mathrm{Dd}}^{\prime \prime} \\
& \text { at } T=T_{\mathrm{H}}, x_{\mathrm{W}}=x_{\mathrm{w}}^{\prime} \text { and } y_{\mathrm{D}}^{\prime}=0 \text { : } \\
& \ln x_{\mathrm{w}}^{\prime}+\ln f_{\mathrm{Hh}}^{\prime}=\ln x_{\mathrm{w}, \mathrm{H}}^{\prime \prime}+\ln f_{\mathrm{Hh}}^{\prime \prime}
\end{aligned}
$$

for $\mu_{\mathrm{II}}$

where $x_{\mathrm{W}}^{\prime}, T_{\mathrm{D}}$ and $x_{\mathrm{W}, \mathrm{D}}^{\prime \prime}, T_{\mathrm{D}}$ are corresponding points on the $\mathrm{D}_{2} \mathrm{O}$-TEA solubility curve and $x_{\mathrm{W}}^{\prime}, T_{\mathrm{H}}$ and $x_{\mathrm{W}, \mathrm{H}}^{\prime \prime}, T_{\mathrm{H}}$, are corresponding points on the $\mathrm{H}_{2} \mathrm{O}$-TEA curve.

By subtraction and introduction of eqn. 42 (integrated)

$$
\left(\ln \frac{f_{\mathrm{Dd}}^{\prime}}{f_{\mathrm{Hh}}}\right)_{\mathrm{T}=\mathrm{T}_{\mathrm{D}}}=\frac{T_{\mathrm{D}}-T_{\mathrm{H}}}{R T_{\mathrm{D}} T_{\mathrm{H}}} \Delta H_{\mathrm{Hh}}^{\prime}+\ln \frac{x_{\mathrm{W}, \mathrm{D}}^{\prime \prime}}{x_{\mathrm{W}, \mathrm{H}}^{\prime \prime}}+\left(\ln f_{\mathrm{Dd}}^{\prime \prime}\right)_{\mathrm{T}=\mathrm{T}_{\mathrm{D}}}-\left(\ln f_{\mathrm{Hh}}^{\prime \prime}\right)_{\mathrm{T}=\mathrm{T}_{\mathrm{H}}}
$$

The vapour pressure data available show that $\ln f_{\mathrm{Hh}}^{\prime \prime} \simeq 0.01$ and it is unlikely that $\ln f_{\mathrm{Dd}}^{\prime \prime}$ is very much different.

Solubility experiments (see Fig. 2) show that

$\ln \frac{x_{\mathrm{W}, \mathrm{D}}^{\prime \prime}}{x_{\mathrm{W}, \mathrm{H}}^{\prime \prime}} \simeq \ln 0.999=-10^{-3}$.

Enthalpy data and solubility data (seo Table 3 and Fig. 2) show that

$$
\frac{T_{\mathrm{D}}-T_{\mathrm{H}}}{R T_{\mathrm{D}} T_{\mathrm{H}}} \Delta H_{\mathrm{Hh}}^{\prime} \simeq 0.03
$$

so eqn. 58 may be reduced to

$$
\left(\ln \frac{f_{\mathrm{Dd}}^{\prime}}{f_{\mathrm{Hh}}}\right)_{\mathrm{T}=\mathrm{T}_{\mathrm{D}}}=\frac{T_{\mathrm{D}}-T_{\mathrm{H}}}{R T_{\mathrm{D}} T_{\mathrm{H}}} \Delta H_{\mathrm{Hh}}^{\prime}
$$


From eqn. 42 it follows that at any temperature, at constant $x_{\mathrm{W}}$

$$
\left(\ln \frac{f_{\mathrm{Dd}}}{f_{\mathrm{Hh}}}\right)_{x_{\mathrm{w}}, T}=-\frac{T-T_{\mathrm{D}}}{R T T_{\mathrm{D}}}\left[\Delta H_{\mathrm{Dd}}-\Delta H_{\mathrm{Hh}}\right]+\frac{T_{\mathrm{D}}-T_{\mathrm{H}}}{R T_{\mathrm{D}} T_{\mathrm{H}}} \Delta H_{\mathrm{Hh}}
$$

which is reduced to eqn. 59 as long as

$$
\left|\left(T-T_{\mathrm{D}}\right)\left(\Delta H_{\mathrm{Dd}}-\Delta H_{\mathrm{Hh}}\right)\right|<<\left|\left(T_{\mathrm{D}}-T_{\mathrm{H}}\right) \Delta H_{\mathrm{Hh}}\right|
$$

This is probably valid in the interval covered by the known parts of the solubility curves. $\Delta H$ has been assumed independent of $T$.

From the reduced form of eqn. 60 it is seen that

$$
\frac{\partial \ln \left(f_{\mathrm{Dd}} / f_{\mathrm{Hh}}\right)}{\partial x_{\mathrm{W}}}=\frac{\mathrm{d}}{\mathrm{d} x_{\mathrm{W}}}\left(\frac{T_{\mathrm{D}}-T_{\mathrm{H}}}{R T_{\mathrm{D}} T_{\mathrm{H}}} \Delta H_{\mathrm{Hh}}\right)
$$

This relationship probably holds even near the critical compositions $\left(x_{\mathrm{cr}, \mathrm{D}}\right.$ may be somewhat different from $x_{\mathrm{cr}, \mathrm{H}}$ )as may be seen in the following way:

$\left(\frac{\mathrm{d} T_{\mathrm{D}}}{\mathrm{d} x_{\mathrm{W}}^{\prime}}\right)_{\mathrm{eq}, y_{\mathrm{D}}^{\prime}=1}$ and $\left(\frac{\mathrm{d} T_{\mathrm{H}}}{\mathrm{d} x_{\mathrm{W}}^{\prime}}\right)_{\mathrm{eq}, y^{\prime}{ }_{\mathrm{D}}=0}$ (subscripts $\mathrm{D}$ and $\mathrm{H}$ refers to the solubility curves for pure $\mathrm{D}_{2} \mathrm{O}$ and $\mathrm{H}_{2} \mathrm{O}$, respectively) are zero at, and very small in an interval around, the critical points. In eqn. 53 the enthalpy terms are proportional to $\left(x_{\mathrm{W}}^{\prime \prime}-x_{\mathrm{W}}^{\prime}\right)^{2}$ near the critical composition as expansion of these terms by Taylor's Series will show so, at $x_{\mathrm{W}} \simeq x_{\mathrm{cr}}$,

$$
\left(\frac{\partial \mu_{\mathrm{Dd}}^{\prime}}{\partial x_{\mathrm{W}}}\right)_{T_{\mathrm{D}}} \simeq\left(\frac{\partial \mu_{\mathrm{Hh}}^{\prime}}{\partial x_{\mathrm{W}}}\right)_{T_{\mathrm{H}}} \simeq 0
$$

this leads to eqn. 62 (cf. eqn. 42) noting that

$$
\frac{\mathrm{d}}{\mathrm{d} x_{\mathrm{W}}}\left(\frac{T_{\mathrm{D}}-T_{\mathrm{H}}}{R T_{\mathrm{D}} T_{\mathrm{H}}}\right) \simeq \frac{1}{R T_{\mathrm{D}} T_{\mathrm{H}}}\left(\frac{\mathrm{d} T_{\mathrm{D}}}{\mathrm{d} x_{\mathrm{W}}}-\frac{\mathrm{d} T_{\mathrm{H}}}{\mathrm{d} x_{\mathrm{W}}}\right) \simeq 0
$$

Incidentally, a comparison of eqns. 63 and 31 shows the limited applicability of the simple approximations, represented by eqn. 25 near the critical points.

3.3.2. Since $f_{\mathrm{Ad}}=f_{\mathrm{Ah}}=1$ for $x_{\mathrm{W}}=0$ (as has been tacitly assumed above) an integration of eqn. 30 at temp. $T$ between 0 and $x_{\mathrm{W}}^{\prime}$ yields

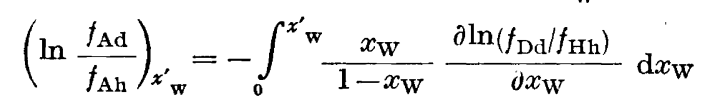

$\lim _{x_{\mathrm{w}} \rightarrow 0} \frac{x_{\mathrm{W}}}{1-x_{\mathrm{W}}} \frac{\partial \ln \left(f_{\mathrm{Dd}} / f_{\mathrm{Hh}}\right)}{\partial x_{\mathrm{W}}}=0$, so extrapolating eqn. 62 to $x_{\mathrm{W}}=0$ and inserting it into eqn. 65 probably results in only a relatively small error:

$$
\left(\ln \frac{f_{\mathrm{Ad}}}{f_{\mathrm{Ah}}}\right)_{x_{\mathrm{W}}^{\prime}} \simeq-\int_{0}^{x^{\prime}{ }_{\mathrm{w}}} \frac{x_{\mathrm{W}}}{1-x_{\mathrm{W}}} \frac{\mathrm{d}}{\mathrm{d} x_{\mathrm{W}}}\left(\frac{T_{\mathrm{D}}-T_{\mathrm{H}}}{R T_{\mathrm{D}} T_{\mathrm{H}}} \Delta H_{\mathrm{Hh}}\right) \mathrm{d} x_{\mathrm{W}}
$$

Integrating by parts

$$
\begin{aligned}
& \left(\ln \frac{f_{\mathrm{Ad}}}{f_{\mathrm{Ah}}}\right)_{x^{\prime}} \simeq-\left[\frac{x_{\mathrm{w}}^{\prime}}{1-x_{\mathrm{W}}^{\prime}} \frac{T_{\mathrm{D}}-T_{\mathrm{H}}}{R T_{\mathrm{D}} T_{\mathrm{H}}} \Delta H_{\mathrm{Hh}}^{\prime}-\int_{0}^{1 /\left(1-x^{\prime}{ }_{\mathrm{w}}^{\prime}\right)} \frac{T_{\mathrm{D}}-T_{\mathrm{H}}}{R T_{\mathrm{D}} T_{\mathrm{H}}} \Delta H_{\mathrm{Hh}} \mathrm{d} \frac{1}{1-x_{\mathrm{W}}}\right] \\
\equiv & -\frac{x_{\mathrm{W}}^{\prime}}{1-x_{\mathrm{W}}^{\prime}}\left[\frac{T_{\mathrm{D}}-T_{\mathrm{H}}}{R T_{\mathrm{D}} T_{\mathrm{H}}} \Delta H_{\mathrm{Hh}}^{\prime}-\frac{T_{\mathrm{D}}-T_{\mathrm{H}}}{R T_{\mathrm{D}} T_{\mathrm{H}}} \Delta H_{\mathrm{Hh}}\right.
\end{aligned}
$$


where $\frac{T_{\mathrm{D}}-T_{\mathrm{H}}}{R T_{\mathrm{D}} T_{\mathrm{H}}} \Delta H_{\mathrm{Hh}}$ is the average of $\frac{T_{\mathrm{D}}-T_{\mathrm{H}}}{R T_{\mathrm{D}} T_{\mathrm{H}}} \Delta H_{\mathrm{Hh}}$ as a function of $1 /\left(1-x_{\mathrm{W}}\right)$ in the interval from 1 to $1 /\left(1-x_{\mathrm{W}}^{\prime}\right)$.

\section{NUMERICAL CALCULATION}

The simplification that the enthalpy of mixing, $\Delta H$, is independent of temperature is not necessary for the development of the system of equations presented above (though a large temperature coefficient would make the step from eqn. 60 to 59 more uncertain). In the numerical calculation, on the other hand, any appreciable change with temperature should be taken into account. Only enthalpy data below $18^{\circ} \mathrm{C}$ are available but it is possible to obtain some idea of the temperature dependence of $\Delta H$ in the range considered from a comparison between results obtained in different ways (see below). The conclusion reached is that $\Delta H$, with sufficient accuracy, may be considered independent of temperature.

First approximations. The simple eqns. 48 or 49 may be used for the calculation of $\left(y_{\mathrm{D}}^{\prime \prime}-y_{\mathrm{D}}^{\prime}\right) / y_{\mathrm{D}}^{\prime}\left(1-y_{\mathrm{D}}^{\prime}\right)$, when $\left(\mathrm{d} T / \mathrm{d} y_{\mathrm{D}}^{\prime}\right)_{\mathrm{eq}, x_{\mathrm{w}}^{\prime}}$ or $\left(\mathrm{d} x_{\mathrm{w}}^{\prime} \mathrm{d} y_{\mathrm{D}}^{\prime}\right)_{\mathrm{eq}, T}$, respectively, are assumed to be constant. These conditions are incompatible and it is seen that $\left(\mathrm{d} x_{\mathrm{w}}^{\prime} / \mathrm{d} y_{\mathrm{D}}^{\prime}\right)_{\text {eq, } T}$ cannot be independent of $y_{\mathrm{D}}^{\prime}$ (at constant $T$ ) near the critical temperature while this is quite possible for $\left(\mathrm{d} T / \mathrm{d} y_{\mathrm{D}}^{\prime}\right)_{\mathrm{eq}, x_{\mathrm{w}}^{\prime}}$ (at constant $\left.x_{\mathrm{w}}^{\prime}\right)$. Eqn. 48 is therefore the more correct. This is, however, of only small importance when the solubility curves are sufficiently close to being straight parallel lines.

Eqn. 49 may be written

where

$$
\frac{y_{\mathrm{D}}^{\prime \prime}-y_{\mathrm{D}}^{\prime}}{y_{\mathrm{D}}^{\prime}\left(1-y_{\mathrm{D}}^{\prime}\right)}=-\frac{x_{\mathrm{w}, \mathrm{d}}^{\prime}-x_{\mathrm{w}, \mathrm{h}}^{\prime}}{2 x_{\mathrm{w}}^{\prime}} Q
$$

$$
Q=\frac{\partial \mu_{\mathrm{w}}^{\prime} / \partial x_{\mathrm{w}}}{R T\left(\partial \ln x_{\mathrm{w}}^{\prime} / \partial x_{\mathrm{w}}\right)}=1+x_{\mathrm{w}}^{\prime} \frac{\partial \ln f_{\mathrm{w}}^{\prime}}{\partial x_{\mathrm{w}}}=x_{\mathrm{w}}^{\prime} \frac{\partial \ln \left(P_{\mathrm{w}}^{\prime} / P_{\mathrm{wo}}\right)}{\partial \ln x_{\mathrm{w}}}
$$

and $x_{\mathrm{W}, \mathrm{h}}^{\prime}$ and $x_{\mathrm{W}, \mathrm{d}}^{\prime}$ are the solubilities of pure $\mathrm{D}_{2} \mathrm{O}$ and $\mathrm{H}_{2} \mathrm{O}$ at temperature $T$. For the present calculation, $Q$ has been found by extrapolating the vapour pressure data of Kohler ${ }^{5}$ at $18^{\circ} \mathrm{C}$ with the help of the enthalpy data from Copp and Everett ${ }^{6}$ at $15^{\circ} \mathrm{C}$ (see Table 3 and Fig. 3 ).

Eqn. 48 may be written

$$
\frac{y_{\mathrm{D}}^{\prime \prime}-y_{\mathrm{D}}^{\prime}}{y_{\mathrm{D}}^{\prime}\left(1-y_{\mathrm{D}}^{\prime}\right)}=\frac{T_{\mathrm{D}}-T_{\mathrm{H}}}{2 R T^{2}} \Delta H_{\mathrm{w}}^{\prime}
$$

where $T_{\mathrm{D}}$ and $T_{\mathrm{H}}$ are the temperatures at which the solubilities of pure $\mathrm{D}_{2} \mathrm{O}$ and $\mathrm{H}_{2} \mathrm{O}$ are $x_{\mathrm{w}}^{\prime}$. Again the $\Delta H$ data from Copp and Everett ${ }^{6}$ have been employed, without any temperature corrections (see Table 3 and Fig. 3).

For both sets of calculations the solubility curves shown in Fig. 2 has been used. These are drawn through the experimental points obtained in this labora- 
Table 3. Numerical results obtained with the vapour pressure data of Kohler ${ }^{5}$, the enthalpy data of Copp and Everett ${ }^{6}$ and with the solubility data shown in Fig. $2 . y_{D}=0.20$. For further explanation see text.

$\begin{array}{rccc}T & -\frac{x_{\mathrm{w}, \mathrm{d}}^{\prime}-x_{\mathrm{W}, \mathrm{h}}^{\prime}}{2 x_{\mathrm{W}}^{\prime}} Q & \frac{T_{\mathrm{D}}-T_{\mathrm{H}}}{2 R T^{2}} \Delta H_{\mathrm{W}}^{\prime} & \frac{T_{\mathrm{D}}-T_{\mathrm{H}}}{2 R T_{\mathrm{D}} T_{\mathrm{H}}} \Delta H_{\mathrm{Hh}} \\ { }^{\circ} \mathrm{C} & \text { (eqn. 68) } & \text { (eqn. 70) } & \text { (eqn. 72) } \\ 22 & 0.008 & 0.009 & 0.018 \\ 27 & 0.017 & 0.013 & 0.025 \\ 35 & 0.025 & 0.021 & 0.029 \\ 45 & 0.022 & 0.025 & 0.030\end{array}$

tory but supported by curves from the paper of Hahn and Street ${ }^{2}$. The absolute position of these curves is not very accurate because small amounts of carbon dioxide may reduce the solubility noticeably (Krichevskii ${ }^{7}$ ).

The agreement at $35^{\circ}$ and $45^{\circ} \mathrm{C}$ between the separation effects based on eqn. 68 and those based on eqn. 70 may be taken to support the assumption used for their calculation that $\Delta H$ varies only little with temperature.

It should be added that such an agreement is not consistent with Kohlers assumption in a later paper ${ }^{8}$ according to which $\Delta H$ should become zero at $70^{\circ} \mathrm{C}$. His conclusion, however, is based on a solubility curve (in a paper by Rothmund ${ }^{9}$ ) which disagrees with others (cf. Fig. 2); in addition, the temperature in question could be anything between $25^{\circ} \mathrm{C}$ and $145^{\circ} \mathrm{C}$, so the disagreement may not be so pronounced as could be expected at first glance.

Second approximations. When $\ln \left(f_{\mathrm{Ad}} / f_{\mathrm{Ah}}\right)$ is taken into account in the calculation of the separation effect eqn. 54 should form the basis. It may be simplified

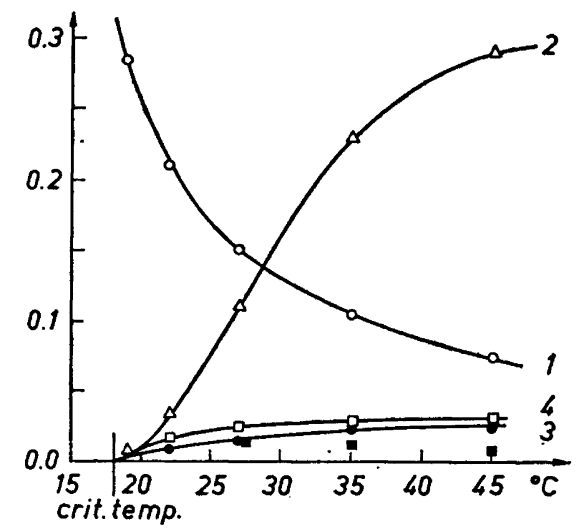

Fig. 3. Calculated (O, eqns. 68 and 70; $\square$, eqn. 72; see Table 3), and experimental ( $\square$ ) isotope separation effects, $\left(y_{\mathrm{D}}^{\prime \prime}-y_{\mathrm{D}}^{\prime}\right) / y_{\mathrm{D}}^{\prime}\left(1-y_{\mathrm{D}}^{\prime}\right)$, as compared to a hypothetic separation between ideal mixtures, $\frac{1}{2}\left(x_{\mathrm{W}, \mathrm{h}}^{\prime}-x_{\mathrm{W}, \mathrm{d}}^{\prime}\right) / x_{\mathrm{W}}^{\prime}$ (O, eqn. 52) and the factor $Q=$ $\left(\partial \mu_{\mathrm{W}}^{\prime} / \partial x_{\mathrm{W}}\right) / R T\left(\partial \ln x_{\mathrm{W}}^{\prime} / \partial x_{\mathrm{W}}\right)(\triangle$, eqn. 69$)$, expressing deviation from ideality. 
since

1) $R T^{2}\left(\partial M^{\prime} / \partial T\right)$ (eqn. 43) is probably small compared to $\Delta H_{\mathrm{Dd}}^{\prime}$ or $\Delta H_{\mathrm{Hh}}^{\prime}$

2) $\Delta H_{\mathrm{Dd}}^{\prime} \simeq \Delta H_{\mathrm{Hh}}^{\prime}$

3) $R T^{2}\left(\partial M^{\prime \prime} \mid \partial T\right) \ll\left\langle\Delta H_{\mathrm{Hh}}^{\prime \prime}\right.$ and $\Delta H_{\mathrm{Dd}}^{\prime \prime} \simeq \Delta H_{\mathrm{Hh}}^{\prime \prime}\left\langle\left\langle\Delta H_{\mathrm{Hh}}^{\prime}\right.\right.$

4) $\left(1-x_{\mathrm{W}}^{\prime \prime}\right)\left(\Delta H_{\mathrm{A}}^{\prime}-\Delta H_{\mathrm{A}}^{\prime \prime}\right)<\left\langle\Delta H_{\mathrm{Hh}}^{\prime}\right.$

5) $x_{\mathrm{w}}^{\prime \prime} \simeq 1$.

In addition the approximation is again made that $\mathrm{d} T / \mathrm{d} y_{\mathrm{D}}^{\prime}=T_{\mathrm{D}}-T_{\mathrm{H}}$. So eqn. 54 becomes

$$
\frac{y_{\mathrm{D}}^{\prime \prime}-y_{\mathrm{D}}^{\prime}}{y_{\mathrm{D}}^{\prime}\left(1-y_{\mathrm{D}}^{\prime}\right)}=\frac{T_{\mathrm{D}}-T_{\mathrm{H}}}{2 R T^{2}} \Delta H_{\mathrm{Hh}}+\frac{1-x_{\mathrm{w}}^{\prime}}{2 x_{\mathrm{W}}^{\prime}}\left(\ln \frac{f_{\mathrm{Ad}}}{f_{\mathrm{Ah}}}\right)_{x^{\prime}{ }_{\mathrm{w}}}
$$

which when eqn. 67 is introduced gives

$$
\frac{y_{\mathrm{D}}^{\prime \prime}-y_{\mathrm{D}}^{\prime}}{y_{\mathrm{D}}^{\prime}\left(1-y_{\mathrm{D}}^{\prime}\right)}=\frac{\overline{1} \frac{T_{\mathrm{D}}-T_{\mathrm{H}}}{2 T_{\mathrm{D}} T_{\mathrm{H}}} \Delta \mathrm{H}_{\mathrm{Hh}}}{2}
$$

(see Table 3 and Fig. 3).

$\overline{\frac{T_{\mathrm{D}}-T_{\mathrm{H}}}{R T_{\mathrm{D}} T_{\mathrm{H}}} \Delta H_{\mathrm{Hh}}}$ has been obtained by graphical integration of

$\frac{T_{\mathrm{D}}-T_{\mathrm{H}}}{R T_{\mathrm{D}} T_{\mathrm{H}}} \Delta H_{\mathrm{Hh}}$ as a function of $\frac{1}{1-x_{\mathrm{W}}}$.

Instead of eqn. 54 it may, however, be more instructive to modify eqn. 55 though the ressulting equation will be less accurate near the critical point, where $\left(\mathrm{d} x_{\mathrm{w}} / \mathrm{d} y_{\mathrm{D}}\right)_{\mathrm{eq}, T} \rightarrow \infty\left(\right.$ while $\left.\left[y_{\mathrm{D}}^{\prime \prime}-y_{\mathrm{D}}^{\prime}\right] /\left[y_{\mathrm{D}}^{\prime}\left(1-y_{\mathrm{D}}^{\prime}\right)\right] \rightarrow 0\right)$ :

If the second-order term

$$
\frac{x_{\mathrm{w}}^{\prime \prime}\left(y_{\mathrm{D}}^{\prime \prime}-y_{\mathrm{D}}^{\prime}\right)}{x_{\mathrm{w}}^{\prime 2}} R T\left(\ln \frac{f_{\mathrm{Ad}}}{f_{\mathrm{Ah}}}\right)_{x_{\mathrm{w}}^{\prime}}\left(\frac{\mathrm{d} x_{\mathrm{w}}^{\prime}}{\mathrm{d} y_{\mathrm{D}}^{\prime}}\right)_{\mathrm{eq}, T}
$$

is neglected and eqn. 34 is introduced, eqn. 55 may be written

$$
\left[\frac{1}{x_{\mathrm{w}}^{\prime}}+y_{\mathrm{D}}^{\prime} \frac{\partial \ln f_{\mathrm{D}}^{\prime}}{\partial x_{\mathrm{W}}}+\left(1-y_{\mathrm{D}}^{\prime}\right) \frac{\partial \ln f_{\mathrm{H}}^{\prime}}{\partial x_{\mathrm{w}}}\right]\left(\frac{\mathrm{d} x_{\mathrm{W}}^{\prime}}{\mathrm{d} y_{\mathrm{D}}^{\prime}}\right)_{\mathrm{eq}, T}=-\frac{\partial \ln f_{\mathrm{H}}^{\prime}}{\partial y_{\mathrm{D}}}-2 \frac{y_{\mathrm{D}}^{\prime \prime}-y_{\mathrm{D}}^{\prime}}{y_{\mathrm{D}}^{\prime}\left(1-y_{\mathrm{D}}^{\prime}\right)}
$$

or, approximately,

$$
\frac{y_{\mathrm{D}}^{\prime \prime}-y_{\mathrm{D}}^{\prime}}{y_{\mathrm{D}}^{\prime}\left(1-y_{\mathrm{D}}^{\prime}\right)} \simeq-\frac{x_{\mathrm{w}, \mathrm{d}}^{\prime}-x_{\mathrm{w}, \mathrm{h}}^{\prime}}{2 x_{\mathrm{w}}^{\prime}}-\frac{x_{\mathrm{w}, \mathrm{d}}^{\prime}-x_{\mathrm{w}, \mathrm{h}}^{\prime}}{2} \frac{\partial \ln f_{\mathrm{H}}^{\prime}}{\partial x_{\mathrm{w}}}-\frac{1}{2} \frac{\partial \ln f_{\mathrm{H}}^{\prime}}{\partial y_{\mathrm{D}}}
$$

(cf. eqns. 68 and 69). The significance of the different terms in eqn. 74 is illustrated in Fig. 3. 


\section{CONCLUSION}

The approximate chemical potential functions and other relations developed above rest on the assumption that the numerical value of $\partial \ln f / \partial y_{\mathrm{D}}$ is small compared to $\partial \ln f / \partial x_{\mathrm{w}}$; this has been verified by calculation, the actual result being

$$
\frac{1}{500} \frac{\partial \ln f_{\mathrm{H}}}{\partial x_{\mathrm{W}}}<\frac{\partial \ln f_{\mathrm{H}}}{\partial y_{\mathrm{D}}}<\frac{1}{100} \frac{\partial \ln f_{\mathrm{H}}}{\partial x_{\mathrm{W}}} \text { in the interval } 0.1<x_{\mathrm{W}}<0.5
$$

In Fig. 3 it is illustrated by the difference between curve 1 and 3 (see Fig. 2 for the numerical value of $x_{\mathrm{w}, \mathrm{d}}^{\prime}-x_{\mathrm{w}, \mathrm{h}}^{\prime}$ ) compared to the difference between curve 3 and 4; see eqn. 74 for further explanation. Curve 1, the relative solubility change divided by the statistical factor, 2 , would be the isotope separation effect to be expected if the simplified version of eqn. 49, analogous to eqn. 52, were applicable - as it would be in an ideal system. The agreement between experimental (also shown in Fig. 3) and theoretical separation effects is satisfactory if the purpose is to verify that this simplification is not permissible.

When the absolute values of $\left[y_{\mathrm{D}}^{\prime \prime}-y_{\mathrm{D}}^{\prime}\right] /\left[y_{\mathrm{D}}^{\prime}\left(1-y_{\mathrm{D}}^{\prime}\right)\right]$ are considered, the discrepancy appears somewhat more important (Note that the experimental values include one or two border line cases).

It may be caused by an erroneous $x^{\prime}{ }_{\mathrm{w}, \mathrm{d}}-x_{\mathrm{w}, \mathrm{h}}^{\prime}$ (eqn. 68); this in fact is the most probable explanation because the effect of introducing $\partial \ln f / \partial y_{\mathrm{D}} \neq 0$, almost certainly must cause an increase in $\left[y_{\mathrm{D}}^{\prime \prime}-y_{\mathrm{D}}^{\prime}\right] /\left[y_{\mathrm{D}}^{\prime}\left(1-y_{\mathrm{D}}^{\prime}\right)\right]$, due to the less ideal appearance of the $\mathrm{D}_{2} \mathrm{O}$-TEA system in comparison to the corresponding $\mathrm{H}_{2} \mathrm{O}$ system.

Expressed in another way: It is probable that $T_{\mathrm{H}}-T_{\mathrm{D}}$ (eqn. 70 ) is too large as the sign of

$$
-\frac{T_{\mathrm{D}}-T_{\mathrm{H}}}{R T_{\mathrm{D}} T_{\mathrm{H}}} \Delta H_{\mathrm{Hh}}^{\prime}+\overline{\frac{T_{\mathrm{D}}-T_{\mathrm{H}}}{R T_{\mathrm{D}} T_{\mathrm{H}}} \Delta H_{\mathrm{Hh}}}
$$

or any modification of this term, representing a better approximation to the non-ideality of the systems, almost certainly, is positive (eqn. 72).

The discrepancy may, however, be caused by impurities not accounted for by chemical analysis. A doubling of the amount found would be sufficient to make the discrepancy disappear. It may be shown, as mentioned earlier, that a trace of water would have the same effect.

From the point of view of deuterium oxide separation it may, in general, be said that the difference between the solubilities of $\mathrm{D}_{2} \mathrm{O}$ and $\mathrm{H}_{2} \mathrm{O}$ in an organic medium $\left(x^{\prime}{ }_{\mathrm{w}, \mathrm{d}}-x^{\prime}{ }_{\mathrm{w}, \mathrm{h}}\right)$ furnishes a very poor measure of the degree of separation obtainable because large $x_{\mathrm{w}, \mathrm{d}}^{\prime}-x_{\mathrm{w}, \mathrm{h}}^{\prime}$ almost invariably must mean that the solubility curve is very flat $\left(\left(\mathrm{d} x_{\mathrm{w}}^{\prime} / \mathrm{d} T\right)_{\mathrm{eq}, y_{\mathrm{D}}}\right.$ large); this again necessitates values of $\left(\partial \mu_{\mathrm{w}}^{\prime} / \partial x_{\mathrm{w}}\right)_{T, y_{\mathrm{D}}}$ very near zero, i.e. very far from the ideal $R T / x_{\mathrm{w}}^{\prime}$, unless the absolute values of $\Delta H$ or/and $T_{\mathrm{D}}-T_{\mathrm{H}}$ are very large. An equation like 68 or 70 is therefore a simple and better basis for comparison.

A general conclusion would seem to be that it is quite unlikely that large $\mathrm{D}_{2} \mathrm{O}-\mathrm{H}_{2} \mathrm{O}$ separation effects in liquid-liquid mixtures are possible, at any rate, in the absence of extensive chemical isotope exchange.

Acta. Chem. Scand. 16 (1962) No. 7 
The author acknowledges with gratitude pern ission to include water-phase solubility determinations obtained by Mr. P. Solgaard.

The author is greatly indebted to Mr. Th. Rosenberg for numerous stimulating discussions and much critical advice.

\section{REFERENCES}

1. Carlson, H. C. Report No. AECD-4085 (1943); U. S. Atomic Energy Comm. Nuclear Sci. Abstr. 10 (1956) 6137.

2. Hahn, H. T. and Street, E. B. Report No. HW 48735 (1957); U. S. Atomic Energy Comm. Nuclear Sci. Abstr. 11 (1957) 6293.

3. Bebbington, W. P., Thayer, V. R. and Proctor, J. F. DP-400 (1959) Appendix A.

4. Kohler, F. Monatsh. 88 (1957) 388.

5. Kohler, F. Monatsh. 82 (1951) 913.

6. Copp, J. L. and Everett, D. H. Discussions Faraday Soc. 1953, No. 15, 174.

7. Krichevskii, I. R., Khazanova, N. E. and Linshitz, L. R. Doklady Akad. Nauk, SSSR 100 (1955) 737.

8. Kohler, F., Arnold, H. and Munn, R. J. Monatsh. 92 (1961) 876.

9. Rothmund, V. Z. physik. Chem. 26 (1898) 461.

10. Lovy, A. L. Nature 174 (1954) 126.

Received March 13, 1962. 